\title{
The Kramer sampling theorem revisited
}

\author{
A. G. García* M. A. Hernández-Medina ${ }^{\dagger}$ and M. J. Muñoz-Bouzo
}

* Departamento de Matemáticas, Universidad Carlos III de Madrid, Avda. de la Universidad 30, 28911 Leganés-Madrid, Spain.

† Departamento de Matemática Aplicada, E.T.S.I.T., U.P.M., Avda. Complutense 30 , 28040 Madrid, Spain.

‡ Departamento de Matemáticas Fundamentales, U.N.E.D., Senda del Rey 9, 28040 Madrid, Spain.

\begin{abstract}
The classical Kramer sampling theorem provides a method for obtaining orthogonal sampling formulas. Besides, it has been the cornerstone for a significant mathematical literature on the topic of sampling theorems associated with differential and difference problems. In this work we provide, in an unified way, new and old generalizations of this result corresponding to various different settings; all these generalizations are illustrated with examples. All the different situations along the paper share a basic approach: the functions to be sampled are obtaining by duality in a separable Hilbert space $\mathcal{H}$ through an $\mathcal{H}$-valued kernel $K$ defined on an appropriate domain.
\end{abstract}

Keywords: Sampling formulas; Kramer kernels; Reproducing kernel Hilbert spaces; Lagrange-type interpolation series; Zero-removing property; Semi-inner products; Reproducing kernel Banach spaces; Reproducing distributions.

AMS: 46E22; 42C15; 94A20.

\section{The classical Kramer sampling theorem}

The classical Kramer sampling theorem provides a method for obtaining orthogonal sampling theorems $[8,13,29,35,48]$. The statement of this general result is as follows. Let $K$ be a complex function defined on $D \times I$, where $I \subset \mathbb{R}$ is an interval and $D$ is an open subset of $\mathbb{R}$, such that for every $t \in D$ the sections $K(\cdot, t)$ are in $L^{2}(I)$. Assume that there exists a sequence of distinct real numbers $\left\{t_{n}\right\} \subset D$, indexed by a subset of $\mathbb{Z}$, such that $\left\{K\left(x, t_{n}\right)\right\}$ is a complete orthogonal sequence of functions for $L^{2}(I)$. Then for any $f$ of the form

$$
f(t)=\int_{I} F(x) K(x, t) d x, \quad t \in D,
$$

\footnotetext{
${ }^{*}$ E-mail:agarcia@math.uc3m.es

${ }^{\dagger}$ E-mail:miguelangel.hernandez.medina@upm.es

${ }^{\ddagger}$ E-mail:mjmunoz@mat . uned.es
} 
where $F \in L^{2}(I)$, we have

$$
f(t)=\sum_{n} f\left(t_{n}\right) S_{n}(t), \quad t \in D,
$$

with

$$
S_{n}(t):=\frac{\int_{I} K(x, t) \overline{K\left(x, t_{n}\right)} d x}{\int_{I}\left|K\left(x, t_{n}\right)\right|^{2} d x}, \quad t \in D .
$$

The series in (1) converges absolutely and uniformly on subsets of $D$ where the function $t \mapsto\|K(\cdot, t)\|_{L^{2}(I)}$ is bounded.

Perhaps, the most important example of the Kramer sampling theorem is the wellknown Whittaker-Shannon-Kotel'nikov sampling result:

Example 1.1. For $I=[-\pi, \pi], D=\mathbb{R}, K(x, t)=\mathrm{e}^{\mathrm{i} t x} / \sqrt{2 \pi}$ and the sequence $\left\{t_{n}=\right.$ $n\}_{n \in \mathbb{Z}}$, we get the WSK sampling formula

$$
f(t)=\sum_{n=-\infty}^{\infty} f(n) \frac{\sin \pi(t-n)}{\pi(t-n)}, \quad t \in \mathbb{R},
$$

for functions $f \in L^{2}(\mathbb{R})$ whose Fourier transform $\widehat{f}$ has support in the interval $[-\pi, \pi]$, i.e., $f(t)=\frac{1}{\sqrt{2 \pi}} \int_{-\pi}^{\pi} \widehat{f}(w) \mathrm{e}^{\mathrm{i} t w} d w, t \in \mathbb{R}$. The series converges absolutely and uniformly on $\mathbb{R}$.

In other words, WSK sampling theorem works for functions $f$ in the classical PaleyWiener space $P W_{\pi}$ of band-limited signals to $[-\pi, \pi]$. See Refs. [9, 18, 27, 28, 32]. Reconstructing integral transforms other than the Fourier one from some sampled values occurs frequently in some physical applications. One such integral transform is the Bessel-Hankel transform. Sampling associated with this transform was first introduced by Kramer in his seminal paper [35]. The Fourier-Bessel set $\left\{\sqrt{x} J_{\nu}\left(x t_{n}\right)\right\}_{n=1}^{\infty}$ is known to be an orthogonal basis for $L^{2}(0,1)$, where $t_{n}$ is the $n$th positive zero of the Bessel function $J_{\nu}(t), \nu>-1$ (see also [29, p. 83]). The Bessel function of order $\nu$ is given by

$$
J_{\nu}(t)=\frac{t^{\nu}}{2^{\nu} \Gamma(\nu+1)}\left[1+\sum_{n=1}^{\infty} \frac{(-1)^{n}}{n !(1+\nu) \cdots(n+\nu)}\left(\frac{t}{2}\right)^{2 n}\right] .
$$

Using special function formulas, for a fixed $t>0$, we have

$$
\sqrt{x t} J_{\nu}(x t)=\sum_{n=1}^{\infty} \frac{2 \sqrt{t_{n} t} J_{\nu}(t)}{J_{\nu}^{\prime}\left(t_{n}\right)\left(t^{2}-t_{n}^{2}\right)} \sqrt{x} J_{\nu}\left(x t_{n}\right), \quad \text { in } L^{2}(0,1) .
$$

Thus, if we take $I=[0,1], D=\mathbb{R}, K(x, t)=\sqrt{x t} J_{\nu}(x t)$ and $\left\{t_{n}\right\}_{n=1}^{\infty}$ the sequence of the positive zeros of $J_{\nu}(t)$ we obtain:

Example 1.2. Any function $f$ of the form $f(t)=\int_{0}^{1} F(x) \sqrt{x t} J_{\nu}(x t) d x, t \in \mathbb{R}$, where $F \in L^{2}(0,1)$, can be recovered from its samples at the positive zeros $\left\{t_{n}\right\}_{n=1}^{\infty}$ of the Bessel function $J_{\nu}$ of $\nu$-th order with $\nu>-1$, through the sampling formula

$$
f(t)=\sum_{n=1}^{\infty} f\left(t_{n}\right) \frac{2 \sqrt{t_{n} t} J_{\nu}(t)}{J_{\nu}^{\prime}\left(t_{n}\right)\left(t^{2}-t_{n}^{2}\right)}, \quad t \in \mathbb{R} .
$$


The Kramer sampling theorem (announced by Weiss in [47]) has been the cornerstone for a significant mathematical literature on the topic of sampling theorems associated with differential or difference problems which has flourished for the past few years. As a small but significant sample of examples see, for instance, [6, 13, 20, 48] and references therein. Here we do not consider this approach.

The main goal in this survey is to give a common framework gathering most of the sampling formulas coming from the Kramer sampling result. The starting point in this paper is an abstract version of the Kramer sampling theorem. To this end, we work in the reproducing kernel Hilbert space (written shortly as RKHS) of functions introduced by Saitoh in [40] as follows: Let $\left(\mathcal{H},\langle\cdot, \cdot\rangle_{\mathcal{H}}\right)$ be a separable Hilbert space with orthonormal basis $\left\{e_{n}\right\}_{n=1}^{\infty}$. Suppose $K$ is a $\mathcal{H}$-valued function defined on $\Omega$ a subset of $\mathbb{R}($ or $\mathbb{C})$. For each $x \in \mathcal{H}$, define $f_{x}(t)=\langle x, K(t)\rangle_{\mathcal{H}}$ and let $\mathcal{H}_{K}$ denote the collection of all such functions $f_{x}$. In this setting, an abstract version of the Kramer theorem is obtained assuming the existence of two sequences, $\left\{t_{n}\right\}_{n=1}^{\infty}$ in $\mathbb{C}$, and $\left\{a_{n}\right\}_{n=1}^{\infty}$ in $\mathbb{C} \backslash\{0\}$, such that $K\left(t_{n}\right)=a_{n} e_{n}$ for each $n \in \mathbb{N}$. This is a slight modification of a sampling result derived by Higgins in [30]. The non-orthogonal case is also included by considering a Riesz basis $\left\{x_{n}\right\}_{n=1}^{\infty}$ instead of the orthonormal basis $\left\{e_{n}\right\}_{n=1}^{\infty}$. Depending on the nature of the Hilbert space $\mathcal{H}$, an $L^{2}$-space, an $\ell^{2}$-space, a Sobolev space, a Hilbert space of distributions, etc., we exhibit different settings where the Kramer sampling theorem applies (Sections 2 and 4). In Section 2 we include the analytic version of Kramer sampling theorem giving a necessary and sufficient condition to ensure when the sampling formula (1) can be expressed as a Lagrange-type interpolation series. We also propose a generalization in Banach spaces by considering a semi-inner-product (Section 3). Finally to say that this work can be seen as a complement to other surveys in the subject [7, 8]; Chapters $4-8$ of Zayed's book [48] are in fact a remarkable survey on Kramer's sampling theorem.

\section{The abstract Kramer sampling theorem}

Since the functions $f$ for which Kramer sampling theorem applies (1) are images of an integral transform with kernel $K$, the reproducing kernel Hilbert spaces considered by Saitoh in [40] are the suitable spaces where a generalization of the classical Kramer sampling theorem also works (see also [41, 45] and [48, Section 10.1]):

\subsection{The RKHS setting}

Let $\mathcal{H}$ be a separable Hilbert space, and $\Omega$ a fixed subset of $\mathbb{R}$. Given an $\mathcal{H}$-valued function $K: \Omega \rightarrow \mathcal{H}$, for any $x \in \mathcal{H}$ the function $f_{x}(t):=\langle x, K(t)\rangle_{\mathcal{H}}, t \in \Omega$, is welldefined as a function $f_{x}: \Omega \rightarrow \mathbb{C}$. We denote by $\mathcal{H}_{K}$ the set of functions obtained in this way and by $\mathcal{T}_{K}$ the linear transform

$$
\mathcal{T}_{K}: \mathcal{H} \ni x \mapsto f_{x} \in \mathcal{H}_{K} .
$$

In other words, $\mathcal{H}_{K}:=\mathcal{T}_{K}(\mathcal{H})$. Hereafter we refer the function $K$ as the kernel of the linear transform $\mathcal{T}_{K}$ and henceforth we omit the subscript $x$ for denoting the elements in $\mathcal{H}_{K}$. Note that the continuity of the kernel $K$ implies that the functions in $\mathcal{H}_{K}$ are 
continuous in $\Omega$. If we define in $\mathcal{H}_{K}$ the norm $\|f\|_{\mathcal{H}_{K}}=\inf \left\{\|x\|_{\mathcal{H}}: f=\mathcal{T}_{K}(x)\right\}$ we obtain a Hilbert space. Moreover, for each $t \in \Omega$, the evaluation functional $E_{t}(f):=$ $f(t), f \in \mathcal{H}_{K}$, is bounded, and the space $\mathcal{H}_{K}$ becomes an RKHS whose reproducing kernel is given by, cf. [40, p. 21],

$$
k(t, s)=\langle K(s), K(t)\rangle_{\mathcal{H}}, \quad t, s \in \Omega,
$$

i.e., for each $s \in \Omega$ the function $k_{s}$ defined as $k_{s}(t):=k(t, s)$ belongs to $\mathcal{H}_{K}$, and the reproducing property

$$
f(s)=\left\langle f, k_{s}\right\rangle_{\mathcal{H}_{K}}=\langle f, k(\cdot, s)\rangle_{\mathcal{H}_{K}}, \quad s \in \Omega, \quad f \in \mathcal{H}_{K},
$$

holds.

In an RKHS space $\mathcal{H}_{K}$, the convergence in the norm $\|\cdot\|_{\mathcal{H}_{K}}$ implies pointwise convergence which is uniform on those subsets of $\Omega$ where the function $t \mapsto\|K(t)\|_{\mathcal{H}}$ is bounded; it follows form (4) by using Cauchy-Schwarz inequality, and having in mind that $k(t, t)=\|K(t)\|^{2}, t \in \Omega$.

Recall that the Moore-Aronszajn procedure [3] leads to the same RKHS via the positive definite (or positive matrix) function $k$ in (3). Under these circumstances it is known that the linear operator $\mathcal{T}_{K}$ is one-to-one if and only if $\mathcal{T}_{K}$ is an isometry between $\mathcal{H}$ and $\mathcal{H}_{K}$, or, equivalently, if and only if the set $\{K(t)\}_{t \in \Omega}$ is complete in $\mathcal{H}$ [40]. The RKHS $\mathcal{H}_{K}$ has been largely studied in the mathematical literature (see Ref. [40] and references therein).

The first generalization consists of stating the Kramer condition in terms of Riesz bases. Recall that a Riesz basis in a separable Hilbert space is the image of an orthonormal basis by means of a bounded invertible operator. Any Riesz basis $\left\{x_{n}\right\}_{n=1}^{\infty}$ has a unique biorthogonal (dual) Riesz basis $\left\{y_{n}\right\}_{n=1}^{\infty}$, i.e., $\left\langle x_{n}, y_{m}\right\rangle_{\mathcal{H}}=\delta_{n, m}$, such that the expansions

$$
x=\sum_{n=1}^{\infty}\left\langle x, y_{n}\right\rangle_{\mathcal{H}} x_{n}=\sum_{n=1}^{\infty}\left\langle x, x_{n}\right\rangle_{\mathcal{H}} y_{n},
$$

hold for every $x \in \mathcal{H}$ (see $[11,46]$ for more details and proofs).

Definition 1 (Abstract Kramer kernel). A kernel $K: \Omega \longrightarrow \mathcal{H}$ is said to be an abstract Kramer kernel with respect to the data $\left\{t_{n}\right\}_{n=1}^{\infty} \in \Omega$ and $\left\{a_{n}\right\}_{n=1}^{\infty} \in \mathbb{C} \backslash\{0\}$ if it satisfies $K\left(t_{n}\right)=\bar{a}_{n} x_{n}, n \in \mathbb{N}$, for some Riesz basis $\left\{x_{n}\right\}_{n=1}^{\infty}$ for $\mathcal{H}$.

For each fixed $t \in \Omega, K(t)$ can be written as $K(t)=\sum_{n=1}^{\infty} \overline{S_{n}(t)} x_{n}$, where the functions

$$
S_{n}(t):=\left\langle y_{n}, K(t)\right\rangle_{\mathcal{H}}, \quad t \in \Omega, \quad n \in \mathbb{N},
$$

belong to $\mathcal{H}_{K}$; here $\left\{y_{n}\right\}_{n=1}^{\infty}$ denotes the dual Riesz basis of $\left\{x_{n}\right\}_{n=1}^{\infty}$ in $\mathcal{H}$. The Kramer condition, $K\left(t_{n}\right)=\bar{a}_{n} x_{n}$ for every $n \in \mathbb{N}$, is equivalent to the interpolatory condition $S_{n}\left(t_{m}\right)=a_{n} \delta_{n, m}, \quad n, m \in \mathbb{N}$.

The Kramer condition also implies that the linear transform (2) is a bijective isometry (unitary operator) between the Hilbert spaces $\mathcal{H}$ and $\mathcal{H}_{K}$. As a consequence, we obtain the following sampling theorem for functions in $\mathcal{H}_{K}$ : 
Theorem 1 (Abstract Kramer sampling theorem). Assume that $K$ is an abstract Kramer kernel for the data $\left\{t_{n}\right\}_{n=1}^{\infty} \in \Omega,\left\{a_{n}\right\}_{n=1}^{\infty} \in \mathbb{C} \backslash\{0\}$ and the Riesz basis $\left\{x_{n}\right\}_{n=1}^{\infty}$ for $\mathcal{H}$. Then, the sequence of functions $\left\{S_{n}\right\}_{n=1}^{\infty}$ given in (5) forms a Riesz basis for the $\mathcal{H}_{K}$. Expanding any $f \in \mathcal{H}_{K}$ in this Riesz basis we obtain the nonorthogonal sampling expansion

$$
f(t)=\sum_{n=1}^{\infty} f\left(t_{n}\right) \frac{S_{n}(t)}{a_{n}}, \quad t \in \Omega .
$$

The series converges in the $\mathcal{H}_{K}$-norm sense and also, absolutely and uniformly on subsets of $\Omega$ where the function $t \mapsto\|K(t)\|_{\mathcal{H}}$ is bounded.

Proof. By (5) we have that $\mathcal{T}_{K}\left(y_{m}\right)=S_{m}$ for each $m \in \mathbb{N}$; since $\mathcal{T}_{K}$ is a bijective isometry we obtain that the sequence $\left\{S_{m}\right\}_{m=1}^{\infty}$ is a Riesz basis for $\mathcal{H}_{K}$ whose biorthogonal basis $\left\{T_{m}\right\}_{m=1}^{\infty}$ is given by $T_{m}:=\mathcal{T}_{K}\left(x_{m}\right), m \in \mathbb{N}$. Expanding any $f \in \mathcal{H}_{K}$ in this Riesz basis, we have $f=\sum_{n=1}^{\infty}\left\langle f, T_{n}\right\rangle_{\mathcal{H}_{K}} S_{n}$, in the $\mathcal{H}_{K}$-norm sense and, consequently, pointwise in $\Omega$. Moreover, having in mind that $\mathcal{T}_{K}$ is an isometry, and that $K\left(t_{n}\right)=\bar{a}_{n} x_{n}$, $n \in \mathbb{N}$, we obtain

$$
\left\langle f, T_{n}\right\rangle_{\mathcal{H}}=\left\langle\mathcal{T}_{K}(x), \mathcal{T}_{K}\left(x_{n}\right)\right\rangle_{\mathcal{H}_{K}}=\left\langle x, x_{n}\right\rangle_{\mathcal{H}}=\left\langle x, K\left(t_{n}\right) / \bar{a}_{n}\right\rangle_{\mathcal{H}}=f\left(t_{n}\right) / a_{n}, \quad n \in \mathbb{N},
$$

and hence the sampling expansion (6). Since a Riesz basis is an unconditional basis (any orthonormal basis is an unconditional basis by using the Parseval equality), the sampling series (6) is pointwise unconditionally convergent for each $t \in \Omega$ and hence pointwise absolutely convergent. The uniform convergence of the series in (6) is a standard result in RKHS's theory.

In the particular case when $\left\{x_{n}\right\}_{n=1}^{\infty}$ is an orthonormal basis for $\mathcal{H}$, it is self-dual and we have the following result:

Corollary 2. Whenever the sequence $\left\{x_{n}\right\}_{n=1}^{\infty}$ in Definition 1 is an orthonormal basis for $\mathcal{H}$, the sequence $\left\{S_{n}\right\}_{n=1}^{\infty}$ is an orthonormal basis for $\mathcal{H}_{K}$ and the sampling expansion (6) is an orthonormal expansion in $\mathcal{H}_{K}$ having the same pointwise convergence properties.

Example 2.1. For $\mathcal{H}:=L^{2}[0, \pi]$ we consider the kernel $K_{c}: \mathbb{R} \longrightarrow L^{2}[0, \pi]$ defined, for each $t \in \mathbb{R}$, by $\left[K_{c}(t)\right](x)=\cos t x, x \in[0, \pi]$. Since the sequence $\{\cos n x\}_{n=0}^{\infty}$ is an orthogonal basis for $L^{2}[0, \pi]$, the kernel $K_{c}$ satisfies the Kramer condition for $n \in$ $\mathbb{N}_{0}:=\mathbb{N} \cup\{0\}$. Thus, any function $f(t)=\langle F, \cos t x\rangle_{L^{2}[0, \pi]}, t \in \mathbb{R}$, where $F \in L^{2}[0, \pi]$, can be expanded as

$$
f(t)=f(0) \frac{\sin \pi t}{\pi t}+\frac{2}{\pi} \sum_{n=0}^{\infty} f(n) \frac{(-1)^{n} t \sin \pi t}{t^{2}-n^{2}}, \quad t \in \mathbb{R} .
$$

Analogously, consider the kernel $K_{s}: \mathbb{R} \longrightarrow L^{2}[0, \pi]$ defined, for each $t \in \mathbb{R}$, by $\left[K_{s}(t)\right](x)=\sin t x, x \in[0, \pi]$. Then, for any function $f(t)=\langle F, \sin t x\rangle_{L^{2}[0, \pi]}, t \in \mathbb{R}$, where $F \in L^{2}[0, \pi]$, the sampling expansion

$$
f(t)=\frac{2}{\pi} \sum_{n=1}^{\infty} f(n) \frac{(-1)^{n} n \sin \pi t}{t^{2}-n^{2}}, \quad t \in \mathbb{R},
$$

holds. The series above converge absolutely and uniformly on $\mathbb{R}$. 


\section{Kramer sampling theorem and indeterminate moment problems}

Discrete Kramer kernels $K$ can be obtained from indeterminate Hamburger moment problem (see Refs. [1, 42] for the details on this theory); here $\mathcal{H}:=\ell^{2}\left(\mathbb{N}_{0}\right)$. Let $s=\left\{s_{n}\right\}_{n \in \mathbb{N}_{0}} \subset \mathbb{R}$ be an indeterminate Hamburger moment sequence, i.e., there exists a non-unique positive Borel measure $\mu$ on $\mathbb{R}$ such that

$$
s_{n}=\int_{-\infty}^{\infty} t^{n} d \mu(t), \quad n \in \mathbb{N}_{0} .
$$

Let $V_{s}$ be the set of positive Borel measures $\mu$ on $\mathbb{R}$ satisfying the moment problem. The functional $\mathcal{L}$ defined on the vector space $\mathbb{C}[x]$ of polynomials $p(t)=\sum_{k=0}^{n} p_{k} t^{k}$ by

$$
\mathcal{L}(p):=\sum_{k=0}^{n} p_{k} s_{k}=\int_{-\infty}^{\infty} p(t) d \mu(t)
$$

is independent of $\mu \in V_{s}$. Let $\left\{P_{n}\right\}_{n=0}^{\infty}$ be the corresponding orthonormal polynomials satisfying

$$
\int_{-\infty}^{\infty} P_{n}(t) P_{m}(t) d \mu(t)=\delta_{n m}, \quad \text { for each } \mu \in V_{s} .
$$

We assume that $P_{n}$ is of degree $n$ with positive leading coefficient. Recall that the sequence $\left\{P_{n}(t)\right\}_{n=0}^{\infty}$ satisfy a three-term recurrence relation

$$
t P_{n}(t)=a_{n} P_{n+1}(t)+b_{n} P_{n}(t)+a_{n-1} P_{n-1}(t), \quad n \geq 0
$$

where $P_{-1}(t)=0$ and $P_{0}(t)=1$. The two sequences $\left\{b_{n}\right\}_{n=0}^{\infty}$ and $\left\{a_{n}\right\}_{n=0}^{\infty}$ of real and positive numbers, respectively, form the semi-infinite Jacobi matrix associated with the indeterminate Hamburger moment problem (see, for instance, [42]). Since we are dealing with an indeterminate Hamburger moment problem it is known that $\sum_{n=0}^{\infty}\left|P_{n}(t)\right|^{2}<\infty$ for each $t \in \mathbb{R}$; in fact, uniformly on compact subsets of $\mathbb{C}$ (see $[1,42])$. Thus, we can consider the kernel

$$
\mathbb{R} \ni t \stackrel{K}{\longmapsto} K(t):=\left\{P_{n}(t)\right\}_{n=0}^{\infty} \in \ell^{2}\left(\mathbb{N}_{0}\right),
$$

and its associated $\mathcal{H}_{K}$ space. Moreover, there exist sequences $\left\{t_{m}\right\}_{m=0}^{\infty}$ in $\mathbb{R}$ such that the sequence $\left\{\left\{P_{0}\left(t_{m}\right), P_{1}\left(t_{m}\right), P_{2}\left(t_{m}\right), \ldots, P_{n}\left(t_{m}\right), \ldots\right\}\right\}_{m=0}^{\infty}$ is an orthogonal basis for $\ell^{2}\left(\mathbb{N}_{0}\right)$ (see [19]). More can be said about these sequences $\left\{t_{m}\right\}_{m=0}^{\infty}$ by using the wellknown Hamburger moment problem theory. An $N$-extremal measure $\mu$ is a solution of the Hamburger moment problem for which the polynomials $\left\{P_{n}\right\}$ are dense in $L^{2}(\mu)$ [42]; the $N$-extremal measures $\mu_{x}$ can be parametrized with $x \in \mathbb{R} \cup\{\infty\}$ and we have that (see [42, p. 126]):

$$
\frac{A(z)+x C(z)}{B(z)+x D(z)}=\int_{-\infty}^{\infty} \frac{d \mu_{x}(y)}{z-y}, \quad z \in \mathbb{C} \backslash \mathbb{R},
$$

where $A(z), B(z), C(z)$ and $D(z)$ are the components of the so-called Nevalinna matrix of the Hamburger indeterminate moment problem (see [42, p. 124]). The sequence of zeros of the entire function, $B(z)+x D(z)$ if $x \in \mathbb{R}$ or the zeros of $D(z)$ if $x=\infty$, are precisely the sampling points $\left\{t_{m}\right\}_{m=0}^{\infty}$ (see $[42$, p. 127]).

As a consequence, associated with any indeterminate Hamburger moment problem we obtain a discrete Kramer kernel $K$. Next we show a concrete example: 
Example 2.2. We consider the particular case of the so-called $q^{-1}$-Hermite polynomials $(0<q<1)$. These polynomials have the explicit representation (see [31])

$$
h_{n}(t \mid q)=\sum_{k=0}^{n} \frac{(q ; q)_{n}}{(q ; q)_{k}(q ; q)_{n-k}}(-1)^{k} q^{k(k-n)}\left(t+\sqrt{t^{2}+1}\right)^{n-2 k},
$$

where the q-shifted factorial notation is used

$$
\left(c_{1}, c_{2}, \cdots, c_{p} ; q\right)_{n}=\prod_{k=1}^{n} \prod_{j=1}^{p}\left(1-c_{j} q^{k-1}\right), \quad \text { for } n=0,1, \ldots, \infty .
$$

The moment problem associated with $\left\{h_{n}(t \mid q)\right\}_{n=0}^{\infty}$ is indeterminate, and the norms of the polynomials are given by $\left\|h_{n}\right\|=\sqrt{\frac{(q ; q)_{n}}{q^{n(n+1) / 2}}}$ (see [31]). In this case the kernel $K_{q}$ is given by

$$
\begin{aligned}
K_{q}: \mathbb{R} & \longrightarrow \ell^{2}\left(\mathbb{N}_{0}\right) \\
t & \longmapsto K_{q}(t):=\left\{P_{n}(t)\right\}_{n=0}^{\infty},
\end{aligned}
$$

where $\left\{P_{n}:=h_{n} /\left\|h_{n}\right\|\right\}_{n=0}^{\infty}$ is the sequence of orthonormal polynomials. Consider, for example, the points $\pm t_{m}:= \pm \frac{1}{2}\left(q^{-m-1 / 2}-q^{m+1 / 2}\right), m \in \mathbb{N}_{0}$, which are the zeros of the corresponding $D$ function, given by (see [31])

$$
D(t)=-\frac{\left(q e^{2 \xi}, q e^{-2 \xi} ; q^{2}\right)_{\infty}}{\left(q ; q^{2}\right)_{\infty}^{2}}, \quad \text { where } t=\sinh \xi
$$

We obtain that

$$
\left\{\left\{P_{0}\left(t_{m}\right), P_{1}\left(t_{m}\right), P_{2}\left(t_{m}\right), \cdots\right\}\right\}_{m \in \mathbb{N}_{0}} \cup\left\{\left\{P_{0}\left(-t_{m}\right), P_{1}\left(-t_{m}\right), P_{2}\left(-t_{m}\right), \cdots\right\}\right\}_{m \in \mathbb{N}_{0}}
$$

is an orthogonal basis for $\ell^{2}\left(\mathbb{N}_{0}\right)$. Following [20], for each $m \in \mathbb{N}_{0}$ we have

$$
S_{m}^{ \pm}(t):=\left\langle\left\{P_{n}\left( \pm t_{m}\right)\right\}_{n \in \mathbb{N}_{0}}, K_{q}(t)\right\rangle_{\ell^{2}\left(\mathbb{N}_{0}\right)}=\frac{D(t)}{\left(t \mp t_{m}\right) D^{\prime}\left( \pm t_{m}\right)}, \quad t \in \mathbb{R}
$$

Thus, Theorem 1 reads: Any function $f$ given by $f(t)=\left\langle\left\{c_{n}\right\},\left\{P_{n}(t)\right\}\right\rangle_{\ell^{2}\left(\mathbb{N}_{0}\right)}, t \in \mathbb{R}$, where $\left\{c_{n}\right\} \in \ell^{2}\left(\mathbb{N}_{0}\right)$, can be expanded as

$$
f(t)=\sum_{m=0}^{\infty} f\left(-t_{m}\right) \frac{D(t)}{\left(t+t_{m}\right) D^{\prime}\left(-t_{m}\right)}+\sum_{m=0}^{\infty} f\left(t_{m}\right) \frac{D(t)}{\left(t-t_{m}\right) D^{\prime}\left(t_{m}\right)}, \quad t \in \mathbb{R} .
$$

The series converges absolutely and uniformly on compact subsets of $\mathbb{R}$.

\subsection{A sampling formula in a shift-invariant space}

In order to avoid most of the drawbacks associated with classical Shannon's sampling theory, sampling and reconstruction problems have been investigated in spline spaces, wavelet spaces, or in general shift-invariant spaces (see [16] and references therein for more details and results on sampling in shift-invariant spaces). 
Let $V_{\varphi}^{2}:=\overline{\operatorname{span}}\{\varphi(\cdot-n)\}_{n \in \mathbb{Z}}$ be a shift-invariant space with stable generator $\varphi \in$ $L^{2}(\mathbb{R})$ which means that the sequence $\{\varphi(\cdot-n)\}_{n \in \mathbb{Z}}$ is a Riesz basis for $V_{\varphi}^{2}$. Recall that the sequence $\{\varphi(\cdot-n)\}_{n \in \mathbb{Z}}$ is a Riesz sequence in $L^{2}(\mathbb{R})$, i.e., a Riesz basis for $V_{\varphi}^{2}$ (see, for instance, [11, p. 143]) if and only if there exist two positive constants $0<A \leq B$ such that

$$
A \leq \sum_{k \in \mathbb{Z}}|\widehat{\varphi}(w+k)|^{2} \leq B, \quad \text { a.e. } w \in[0,1]
$$

where $\widehat{\varphi}$ stands for the Fourier transform of $\varphi$ (defined as $\widehat{\varphi}(w):=\int_{-\infty}^{\infty} \varphi(t) \mathrm{e}^{-2 \pi \mathrm{i} w t} d t$ in $\left.L^{1}(\mathbb{R}) \cap L^{2}(\mathbb{R})\right)$. Thus we have that

$$
V_{\varphi}^{2}=\left\{\sum_{n \in \mathbb{Z}} a_{n} \varphi(\cdot-n):\left\{a_{n}\right\} \in \ell^{2}(\mathbb{Z})\right\} \subset L^{2}(\mathbb{R}) .
$$

We assume that the functions in the shift-invariant space $V_{\varphi}^{2}$ are continuous on $\mathbb{R}$. This is equivalent to say that the generator $\varphi$ is continuous on $\mathbb{R}$ and the function $t \mapsto \sum_{n \in \mathbb{Z}}|\varphi(t-n)|^{2}$ is bounded on $\mathbb{R}$. Thus, any $f \in V_{\varphi}^{2}$ is defined on $\mathbb{R}$ as the pointwise sum $f(t)=\sum_{n \in \mathbb{Z}} a_{n} \varphi(t-n)$ for each $t \in \mathbb{R}$.

On the other hand, the space $V_{\varphi}^{2}$ is the image of the Hilbert space $L^{2}[0,1]$ by means of the isomorphism

$$
\begin{gathered}
\mathcal{T}_{\varphi}: \begin{array}{c}
L^{2}[0,1] \\
\left\{\mathrm{e}^{-2 \pi i n x}\right\}_{n \in \mathbb{Z}}
\end{array} \quad \longmapsto\{\varphi(t-n)\}_{n \in \mathbb{Z}},
\end{gathered}
$$

which maps the orthonormal basis $\left\{\mathrm{e}^{-2 \pi i n w}\right\}_{n \in \mathbb{Z}}$ for $L^{2}[0,1]$ onto the Riesz basis $\{\varphi(t-$ $n)\}_{n \in \mathbb{Z}}$ for $V_{\varphi}^{2}$. For any $F \in L^{2}[0,1]$ we have

$$
\mathcal{T}_{\varphi} F(t)=\sum_{n \in \mathbb{Z}}\left\langle F, \mathrm{e}^{-2 \pi i n x}\right\rangle \varphi(t-n)=\left\langle F, \sum_{n \in \mathbb{Z}} \overline{\varphi(t-n)} \mathrm{e}^{-2 \pi i n x}\right\rangle=\left\langle F, K_{t}\right\rangle_{L^{2}[0,1]}, \quad t \in \mathbb{R},
$$

where, for each $t \in \mathbb{R}$, the function $K_{t} \in L^{2}[0,1]$ is given by

$$
K_{t}(x):=\sum_{n \in \mathbb{Z}} \overline{\varphi(t-n)} \mathrm{e}^{-2 \pi i n x}=\overline{\sum_{n \in \mathbb{Z}} \varphi(t+n) \mathrm{e}^{-2 \pi i n x}}=\overline{Z \varphi(t, x)} .
$$

Here, $Z \varphi(t, x):=\sum_{n \in \mathbb{Z}} \varphi(t+n) \mathrm{e}^{-2 \pi i n x}$ is just the Zak transform of the function $\varphi$ (see [11] for properties and uses of the Zak transform).

As a consequence, the shift-invariant space $V_{\varphi}^{2}$ is an RKHS in $L^{2}(\mathbb{R})$, and any function $f=\mathcal{T}_{\varphi} F \in V_{\varphi}^{2}$ can be expressed as

$$
f(t)=\left\langle F, K_{t}\right\rangle_{L^{2}[0,1]}, \quad t \in \mathbb{R} .
$$

Thus, for $a \in[0,1)$ fixed and $m \in \mathbb{Z}$ we have

$$
f(a+m)=\left\langle F, K_{a+m}\right\rangle_{L^{2}[0,1]}=\left\langle F, \mathrm{e}^{-2 \pi i m x} K_{a}\right\rangle_{L^{2}[0,1]}, \quad F=\mathcal{T}_{\varphi}^{-1} f .
$$

In order to apply Theorem 1, we look for sampling points of the form $t_{m}:=a+m$, $m \in \mathbb{Z}$, such that the sequence $\left\{\mathrm{e}^{-2 \pi i m x} K_{a}(x)\right\}_{m \in \mathbb{Z}}$ is a Riesz basis for $L^{2}[0,1]$.

Recalling that the multiplication operator $m_{F}: L^{2}[0,1] \rightarrow L^{2}[0,1]$ given as the product $m_{F}(f)=F f$ is well-defined if and only if $F \in L^{\infty}[0,1]$, and then, it is bounded with norm $\left\|m_{F}\right\|=\|F\|_{\infty}$, the following result comes out: 
Lemma 3. The sequence of functions $\left\{\mathrm{e}^{-2 \pi i m x} K_{a}(x)\right\}_{m \in \mathbb{Z}}$ is a Riesz basis for $L^{2}[0,1]$ if and only if the inequalities $0<\left\|K_{a}\right\|_{0} \leq\left\|K_{a}\right\|_{\infty}<\infty$ hold, where $\left\|K_{a}\right\|_{0}:=$

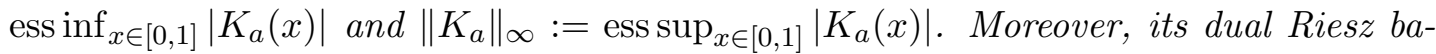
sis is $\left\{\mathrm{e}^{-2 \pi i m x} / \overline{K_{a}(x)}\right\}_{m \in \mathbb{Z}}$. In particular, the sequence $\left\{\mathrm{e}^{-2 \pi i m x} K_{a}(x)\right\}_{m \in \mathbb{Z}}$ is an orthonormal basis in $L^{2}[0,1]$ if and only if $\left|K_{a}(x)\right|=1$ a.e. in $[0,1]$.

Let $a$ be a real number in $[0,1)$ such that $0<\left\|K_{a}\right\|_{0} \leq\left\|K_{a}\right\|_{\infty}<\infty$. For each $n \in \mathbb{N}$ we have

$$
S_{n}(t):=\left\langle\mathrm{e}^{-2 \pi i n x} / \overline{K_{a}(x)}, K_{t}(x)\right\rangle_{L^{2}[0,1]}=\mathcal{T}_{\varphi}\left(\mathrm{e}^{-2 \pi i n x} / \overline{K_{a}(x)}\right)(t)=S_{a}(t-n), \quad t \in \mathbb{R},
$$

where $S_{a}:=\mathcal{T}_{\varphi}\left(1 / \overline{K_{a}}\right) \in V_{\varphi}^{2}$, and we have used the shifting property $\mathcal{T}_{\varphi}\left(\mathrm{e}^{-2 \pi i n x} F\right)(t)=$ $\left(\mathcal{T}_{\varphi} F\right)(t-n), t \in \mathbb{R}$ and $n \in \mathbb{Z}$, which satisfies $\mathcal{T}_{\varphi}$. As a consequence, Theorem 1 reads: Any function $f \in V_{\varphi}^{2}$ can be expanded as the sampling series

$$
f(t)=\sum_{n=-\infty}^{\infty} f(a+n) S_{a}(t-n), \quad t \in \mathbb{R} .
$$

The convergence of the series in (8) is absolute and uniform on $\mathbb{R}$ since the function $t \mapsto\left\|K_{t}\right\|^{2}=\sum_{n \in \mathbb{Z}}|\varphi(t-n)|^{2}$ is bounded on $\mathbb{R}$.

Important examples of shift-invariant spaces $V_{\varphi}^{2}$ are those generated by $B$-splines:

Example 2.3. Consider $\varphi:=N_{m}$ where $N_{m}$ is the B-spline of order $m-1$, i.e., $N_{m}:=N_{1} * N_{1} * \cdots * N_{1}$ (m times) where $N_{1}:=\chi_{[0,1]}$ denotes the characteristic function of the interval $[0,1]$. It is known that the sequence $\left\{N_{m}(t-n)\right\}_{n \in \mathbb{Z}}$ is a Riesz basis for $V_{N_{m}}^{2}$ (see, for instance, [11]). For example, the following sampling formulas hold:

(1) For any $f \in V_{N_{2}}^{2}$, it is obvious that $f(t)=\sum_{n=-\infty}^{\infty} f(n) N_{2}(t+1-n), t \in \mathbb{R}$.

(2) For the quadratic spline $N_{3}$ we have $Z N_{3}(t, x)=\frac{t^{2}}{2}+\left[\frac{3}{4}-\left(t-\frac{1}{2}\right)^{2}\right] z+\frac{(1-t)^{2}}{2} z^{2}$ where $z=\mathrm{e}^{-2 \pi i x}$. Thus, for $t=0$ we have $Z N_{3}(0, x)=\frac{z}{2}(1+z)$ which vanishes at $x=1 / 2$. However, for $t=1 / 2$ we have $Z N_{3}(1 / 2, x)=\frac{1}{8}\left(1+6 z+z^{2}\right)$; according to $(7)$ we deduce $0<\left\|K_{1 / 2}\right\|_{0} \leq\left\|K_{1 / 2}\right\|_{\infty}<\infty$. Hence, for any $f \in V_{N_{3}}^{2}$, we have

$$
f(t)=\sum_{n=-\infty}^{\infty} f\left(n+\frac{1}{2}\right) S_{1 / 2}(t-n), \quad t \in \mathbb{R},
$$

where $S_{1 / 2}(t)=\sqrt{2} \sum_{n=-\infty}^{\infty}(2 \sqrt{2}-3)^{|n+1|} N_{3}(t-n)$. This function has been obtained from the Laurent expansion of the function $8\left(1+6 z+z^{2}\right)^{-1}$ in the annulus $3-2 \sqrt{2}<$ $|z|<3+2 \sqrt{2}$.

(3) Since $Z N_{4}(0, x)=\frac{z}{6}\left(1+4 z+z^{2}\right)=\frac{z}{6}(z-\lambda)(z-1 / \lambda)$ where $z=\mathrm{e}^{-2 \pi i x}$ and $\lambda=\sqrt{3}-2$, according to (7) we deduce that $0<\left\|K_{0}\right\|_{0} \leq\left\|K_{0}\right\|_{\infty}<\infty$. Thus, for any $f \in V_{N_{4}}^{2}$ we have

$$
f(t)=\sum_{n=-\infty}^{\infty} f(n) S_{0}(t-n), \quad t \in \mathbb{R},
$$


where $S_{0}(t)=\sqrt{3} \sum_{n=-\infty}^{\infty}(-1)^{n}(2-\sqrt{3})^{|n|} N_{4}(t-n+2)$. To obtain the function $S_{0}$ we have used the Laurent expansion of the function $6\left(z+4 z^{2}+z^{3}\right)^{-1}$ in the annulus $2-\sqrt{3}<|z|<2+\sqrt{3}$.

\subsection{The analytic version}

Suppose that $\Omega=\mathbb{C}$ and let $K$ be an analytic $\mathcal{H}$-valued function defined on $\mathbb{C}$ where $\mathcal{H}$ denotes a separable complex Hilbert space. Set $f_{x}(z):=\langle K(z), x\rangle_{\mathcal{H}}, z \in \mathbb{C}$, and denote by $\mathcal{H}_{K}$ the collection of all such functions $f_{x}, x \in \mathcal{H}$. We place $K$ in the first argument of the inner product in order to transfer the analyticity of $K$ to the functions of $\mathcal{H}_{K}$. In fact, $K$ is an analytic $\mathcal{H}$-valued function defined on $\mathbb{C}$ if and only if every $f_{x} \in \mathcal{H}_{K}$ is an entire function $\left([43\right.$, p. 266] $)$. As in the previous case, the space $\mathcal{H}_{K}$ is a reproducing kernel Hilbert space with reproducing kernel $k(z, w)=\langle K(z), K(w)\rangle_{\mathcal{H}}$, $z, w \in \mathbb{C}$. Notice that the mapping $\mathcal{T}_{K}$ given by

$$
\mathcal{H} \ni x \stackrel{\mathcal{T}_{K}}{\longmapsto} f_{x} \in \mathcal{H}_{K}
$$

is an anti-linear mapping from $\mathcal{H}$ onto $\mathcal{H}_{K}$. Thus, the space $\mathcal{H}_{K}$ is an RKHS of entire functions. Another characterization of the analyticity of the functions in $\mathcal{H}_{K}$ is given in terms of Riesz bases by using Montel's theorem. Indeed, suppose that a Riesz basis $\left\{x_{n}\right\}_{n=1}^{\infty}$ for $\mathcal{H}$ is given and let $\left\{y_{n}\right\}_{n=1}^{\infty}$ be its dual Riesz basis; expanding $K(z)$, for each fixed $z \in \mathbb{C}$, with respect to the basis $\left\{x_{n}\right\}_{n=1}^{\infty}$ we obtain

$$
K(z)=\sum_{n=1}^{\infty}\left\langle K(z), y_{n}\right\rangle_{\mathcal{H}} x_{n},
$$

where the coefficients $\left\langle K(z), y_{n}\right\rangle_{\mathcal{H}}$, as functions in $z$, are in $\mathcal{H}_{K}$. The following result holds (see [21] for the proof):

Lemma 4. The space $\mathcal{H}_{K}$ is an RKHS of entire functions if and only if for each $n \in \mathbb{N}$ the function

$$
S_{n}(z):=\left\langle K(z), y_{n}\right\rangle_{\mathcal{H}}, \quad z \in \mathbb{C},
$$

is entire, and the function $z \mapsto\|K(z)\|_{\mathcal{H}}$ is bounded on compact subsets of $\mathbb{C}$.

Now, according to Definition 1 the analytic kernel $K: \mathbb{C} \longrightarrow \mathcal{H}$ is said to be an analytic Kramer kernel with respect to the data $\left\{z_{n}\right\}_{n=1}^{\infty} \in \mathbb{C}$ and $\left\{a_{n}\right\}_{n=1}^{\infty} \in \mathbb{C} \backslash\{0\}$ if it satisfies $K\left(z_{n}\right)=a_{n} x_{n}, n \in \mathbb{N}$, for some Riesz basis $\left\{x_{n}\right\}_{n=1}^{\infty}$ for $\mathcal{H}$.

An analytic kernel $K$ is an analytic Kramer one if and only if the sequence of functions $\left\{S_{n}\right\}_{n=1}^{\infty}$ in $\mathcal{H}_{K}$ given by (10), where $\left\{y_{n}\right\}_{n=1}^{\infty}$ is the dual Riesz basis of $\left\{x_{n}\right\}_{n=1}^{\infty}$ satisfies the interpolation property $S_{n}\left(z_{m}\right)=a_{n} \delta_{n, m}, m, n \in \mathbb{N}$. As a consequence, $\lim _{n \rightarrow \infty}\left|z_{n}\right|=+\infty$; otherwise the sequence $\left\{z_{n}\right\}_{n=1}^{\infty}$ contains a bounded subsequence and hence, the entire function $S_{n} \equiv 0$ for all $n \in \mathbb{N}$ which contradicts that $S_{n}\left(z_{n}\right)=a_{n}$ for each $n \in \mathbb{N}$.

Concerning the existence of analytic Kramer kernels, it has been proved in [22] that, associated with any arbitrary sequence of complex numbers $\left\{z_{n}\right\}_{n=1}^{\infty}$ such that $\lim _{n \rightarrow \infty}\left|z_{n}\right|=+\infty$, there exists an analytic Kramer kernel $K$.

Under the notation introduced so far a slight modification of Theorem 1 holds: 
Theorem 5 (Analytic Kramer sampling theorem). Let $K: \mathbb{C} \longrightarrow \mathcal{H}$ be an analytic Kramer kernel with respect to the data $\left\{z_{n}\right\}_{n=1}^{\infty} \in \mathbb{C}$ and $\left\{a_{n}\right\}_{n=1}^{\infty} \in \mathbb{C} \backslash\{0\}$. Let $\mathcal{H}_{K}$ be the corresponding RKHS of entire functions. Then any $f \in \mathcal{H}_{K}$ can be recovered from its samples $\left\{f\left(z_{n}\right)\right\}_{n=1}^{\infty}$ by means of the sampling series

$$
f(z)=\sum_{n=1}^{\infty} f\left(z_{n}\right) \frac{S_{n}(z)}{a_{n}}, \quad z \in \mathbb{C},
$$

where the reconstruction functions $S_{n}$ are given in (10). The series converges absolutely and uniformly on compact subsets of $\mathbb{C}$.

Proof. The anti-linear mapping $\mathcal{T}_{K}$ given by (9) is a bijective isometry between $\mathcal{H}$ and $\mathcal{H}_{K}$. As a consequence, the functions $\left\{S_{n}=\mathcal{T}_{K}\left(y_{n}\right)\right\}_{n=1}^{\infty}$ form a Riesz basis for $\mathcal{H}_{K}$; let $\left\{T_{n}\right\}_{n=1}^{\infty}$ be its dual Riesz basis. Expanding any $f \in \mathcal{H}_{K}$ in this basis we obtain $f=\sum_{n=1}^{\infty}\left\langle f, T_{n}\right\rangle_{\mathcal{H}_{K}} S_{n}$ in $\mathcal{H}_{K}$. Moreover,

$$
\left\langle f, T_{n}\right\rangle_{\mathcal{H}_{K}}={\overline{\left\langle x, x_{n}\right\rangle_{\mathcal{H}}}}=\left\langle K\left(z_{n}\right) / a_{n}, x\right\rangle_{\mathcal{H}}=f\left(z_{n}\right) / a_{n}, \quad n \in \mathbb{N} .
$$

Since a Riesz basis is an unconditional basis, the sampling series will be pointwise unconditionally convergent and hence, absolutely convergent. The uniform convergence is a standard result in the setting of the RKHS theory since the function $z \mapsto\|K(z)\|_{\mathcal{H}}$ is bounded on compact subsets of $\mathbb{C}$.

Let $\left\{P_{n}(z)\right\}_{n=0}^{\infty}$ be a sequence of orthonormal polynomials associated with an indeterminate Hamburger moment problem. It is known (see $[1,42])$ that the series $\sum_{n=0}^{\infty}\left|P_{n}(z)\right|^{2}<\infty$, uniformly on compact subsets of $\mathbb{C}$. Thus, we can define a discrete analytic kernel $K$ as

$$
\mathbb{C} \ni z \stackrel{K}{\longmapsto} K(z):=\left\{P_{n}(z)\right\}_{n=0}^{\infty} \in \ell^{2}\left(\mathbb{N}_{0}\right) .
$$

Let $\mathcal{H}_{K}$ be its corresponding RKHS of entire functions; any $f \in \mathcal{H}_{K}$ has the form

$$
f(z)=\left\langle\left\{P_{n}(z)\right\},\left\{c_{n}\right\}\right\rangle_{\ell^{2}\left(\mathbb{N}_{0}\right)}, \quad z \in \mathbb{C},
$$

where $\left\{c_{n}\right\} \in \ell^{2}\left(\mathbb{N}_{0}\right)$. For the sequences $\left\{t_{m}\right\}_{m=0}^{\infty}$ introduced in Section 2.1, the kernel $K$ also becomes an analytic Kramer kernel. Next we exhibit an example taken from polynomials associated with birth and death processes (see the classical Ref. [33]):

Example 2.4. Consider birth and death polynomials $\left\{P_{n}\right\}_{n \in \mathbb{N}_{0}}$ with quartic rates [5]. These polynomials satisfy the three-term recurrence relation

$$
\begin{gathered}
x P_{n}(x)=a_{n} P_{n+1}(x)+b_{n} P_{n}(x)+a_{n-1} P_{n-1}(x), \quad n \geq 0 \\
P_{-1}(x)=0 ; \quad P_{0}(x)=1,
\end{gathered}
$$

where, for $n \geq 0$, the coefficients are given by

$$
\begin{array}{ll}
a_{n}=\sqrt{\lambda_{n} \mu_{n+1}} & b_{n}=\lambda_{n}+\mu_{n} \\
\lambda_{n}=(4 n+1)(4 n+2)^{2}(4 n+3) & \mu_{n}=(4 n-1)(4 n)^{2}(4 n+1)
\end{array}
$$


By Favard's theorem these polynomials are orthonormal polynomials with respect to a normalized Hamburger moment sequence $s$ which is indeterminate [5]. The entire function $D$ in Nevanlinna parametrization is given by

$$
D(z)=\frac{4}{\pi} \sqrt{z} \sin \left(\frac{\sqrt[4]{z}}{2} K_{0}\right) \sinh \left(\frac{\sqrt[4]{z}}{2} K_{0}\right), \quad z \in \mathbb{C},
$$

whose zeros are $z_{m}=\left(2 \pi m / K_{0}\right)^{4}, m \in \mathbb{N}_{0}$, and $K_{0}$ denotes the constant $\frac{\Gamma^{2}(1 / 4)}{4 \sqrt{\pi}}[5]$. For each $m \in \mathbb{N}_{0}$ we have [5, 20]:

$$
S_{m}(z):=\left\langle K(z),\left\{P_{n}\left(z_{m}\right)\right\}_{n=0}^{\infty}\right\rangle_{\ell^{2}\left(\mathbb{N}_{0}\right)}=\frac{D(z)}{\left(z-z_{m}\right) D^{\prime}\left(z_{m}\right)}, \quad z \in \mathbb{C} .
$$

Hence, any entire function of the form $f(z)=\left\langle K(z),\left\{c_{n}\right\}\right\rangle_{\ell^{2}\left(\mathbb{N}_{0}\right)}=\sum_{n=0}^{\infty} \bar{c}_{n} P_{n}(z)$, $z \in \mathbb{C}$, where $\left\{c_{n}\right\} \in \ell^{2}\left(\mathbb{N}_{0}\right)$ can be expanded as

$$
\begin{aligned}
f(z) & =f(0) \frac{4 \sqrt{z} \sin \left(\frac{\sqrt[4]{z}}{2} K_{0}\right) \sinh \left(\frac{\sqrt[4]{z}}{2} K_{0}\right)}{z K_{0}^{2}}+ \\
& +\frac{16 \pi}{K_{0}^{2}} \sum_{m=1}^{\infty} f\left[\left(\frac{2 \pi m}{K_{0}}\right)^{4}\right] \frac{(-1)^{m} m}{\sinh (m \pi)} \frac{\sqrt{z} \sin \left(\frac{\sqrt[4]{z}}{2} K_{0}\right) \sinh \left(\frac{\sqrt[4]{z}}{2} K_{0}\right)}{\left(z-\left(\frac{2 \pi m}{K_{0}}\right)^{4}\right)}, z \in \mathbb{C} .
\end{aligned}
$$

The series converges absolutely and uniformly on compact subsets of $\mathbb{C}$.

We have used that

$$
D^{\prime}(0)=\frac{K_{0}^{2}}{\pi} \quad \text { and } \quad D^{\prime}\left[\left(\frac{2 \pi m}{K_{0}}\right)^{4}\right]=\frac{K_{0}^{2}}{4 m \pi^{2}}(-1)^{m} \sinh (m \pi) .
$$

Example 2.5. Let $H^{1}(-\pi, \pi)$ be the Sobolev Hilbert space with its usual inner product

$$
\langle f, g\rangle_{1}:=\int_{-\pi}^{\pi} f(x) \overline{g(x)} d x+\int_{-\pi}^{\pi} f^{\prime}(x) \overline{g^{\prime}(x)} d x, \quad f, g \in H^{1}(-\pi, \pi) .
$$

The system $\left\{e^{i n x}\right\}_{n \in \mathbb{Z}} \cup\{\sinh x\}$ forms an orthogonal basis for $H^{1}(-\pi, \pi)$ : It is easy to prove that the orthogonal complement of $\left\{e^{i n x}\right\}_{n \in \mathbb{Z}}$ in $H^{1}(-\pi, \pi)$ is one-dimensional and $\sinh x$ belongs to it. Given $a \in \mathbb{C} \backslash \mathbb{Z}$ we define a kernel $K_{a}: \mathbb{C} \longrightarrow H^{1}(-\pi, \pi)$ by setting

$$
\left[K_{a}(z)\right](x)=(z-a) e^{i z x}+\sin \pi z \sinh x, \quad x \in(-\pi, \pi) .
$$

Expanding $K_{a}(z) \in H^{1}(-\pi, \pi)$ with respect to the above orthogonal basis we obtain

$K_{a}(z)=[1-i(z-a)] \sin \pi z \sinh x+(z-a) \sum_{n=-\infty}^{\infty} \frac{1+z n}{1+n^{2}} \operatorname{sinc}(z-n) e^{i n x}$ in $H^{1}(-\pi, \pi)$,

where $\operatorname{sinc} z=\sin \pi z / \pi z$ denotes the cardinal sine function. As a consequence, Theorem 5 reads: Any entire function $f$ given by

$$
f(z)=\int_{-\pi}^{\pi} F(x)\left[K_{a}(z)\right](x) d x+\int_{-\pi}^{\pi} F^{\prime}(x)\left[K_{a}(z)\right]^{\prime}(x) d x=\left\langle K_{a}(z), \bar{F}\right\rangle_{1}, \quad z \in \mathbb{C},
$$


where $F \in H^{1}(-\pi, \pi)$, can be recovered from its samples $\{f(n)\}_{n \in \mathbb{Z}} \cup\{f(a)\}$ by means of the sampling formula

$$
f(z)=[1-i(z-a)] \frac{\sin \pi z}{\sin \pi a} f(a)+\sum_{n=-\infty}^{\infty} f(n) \frac{z-a}{n-a} \frac{1+z n}{1+n^{2}} \operatorname{sinc}(z-n), \quad z \in \mathbb{C} .
$$

The series converges absolutely and uniformly on compact subsets of $\mathbb{C}$.

\section{Analytic Kramer kernels and Lagrange-type interpolation series}

A more difficult question concerns whether the sampling expansion (11) can be written, in general, as a Lagrange-type interpolation series. Note that, for any $f \in P W_{\pi}$, the corresponding sampling formula can be written as:

$$
f(z)=\sum_{n=-\infty}^{\infty} f(n) \frac{\sin \pi(z-n)}{\pi(z-n)}=\sum_{n=-\infty}^{\infty} f(n) \frac{P(z)}{P^{\prime}(n)(z-n)}, \quad z \in \mathbb{C},
$$

where $P(z)=\sin \pi z$ is an entire function having simple zeros at $\mathbb{Z}$. A complete answer to this question involves the following algebraic property:

Definition 2. A space $\mathcal{H}$ of entire functions has the zero-removing property (ZR property hereafter) if for any $g \in \mathcal{H}$ and any zero $w$ of $g$ the function $g(z) /(z-w)$ belongs to $\mathcal{H}$.

The classical Paley-Wiener space $P W_{\pi}:=\left\{f \in L^{2}(\mathbb{R}) \cap C(\mathbb{R})\right.$, supp $\left.\widehat{f} \subseteq[-\pi, \pi]\right\}$, where $\widehat{f}$ stands for the Fourier transform of $f$, satisfies the ZR property. It follows, for instance, from its characterization by using the classical Paley-Wiener theorem [46, p.101], i.e.,

$$
P W_{\pi}=\left\{f \text { entire function }:|f(z)| \leq A \mathrm{e}^{\pi|z|},\left.\quad f\right|_{\mathbb{R}} \in L^{2}(\mathbb{R})\right\} .
$$

Other examples where the ZR property holds can be found in [15]. There exists a necessary and sufficient condition to ensure when the sampling formula (11) associated with an analytic Kramer kernel can be expressed as a Lagrange-type interpolation series (see Refs. [14, 15] for the proof):

Theorem 6. Let $\mathcal{H}_{K}$ be an RKHS of entire functions obtained from an analytic Kramer kernel $K$ with respect to the sequence $\left\{z_{n}\right\}_{n=1}^{\infty} \subset \mathbb{C}$. Then, the sampling formula (11) for $\mathcal{H}_{K}$ can be written as a Lagrange-type interpolation series

$$
f(z)=\sum_{n=-\infty}^{\infty} f\left(z_{n}\right) \frac{P(z)}{\left(z-z_{n}\right) P^{\prime}\left(z_{n}\right)}, \quad z \in \mathbb{C},
$$

where $P$ denotes an entire function having simple zeros at $\left\{z_{n}\right\}$ if and only if the space $\mathcal{H}_{K}$ satisfies the $Z R$ property. 


\section{Example 2.6. (The Paley-Wiener-Levinson theorem revisited)}

Let $\left\{z_{n}\right\}_{n \in \mathbb{Z}}$ be a sequence in $\mathbb{C}$ satisfying $\sup _{n}\left|\operatorname{Re} z_{n}-n\right|<1 / 4$ and $\sup _{n}\left|\operatorname{Im} z_{n}\right|<\infty$. It is known that the system $\left\{\mathrm{e}^{i z_{n} w} / \sqrt{2 \pi}\right\}_{n \in \mathbb{Z}}$ is a Riesz basis for $L^{2}[-\pi, \pi]$ (see [46, p. 196]). The Fourier kernel $\mathbb{C} \ni z \mapsto K(z)=\mathrm{e}^{\mathrm{i} z} \cdot / \sqrt{2 \pi} \in L^{2}[-\pi, \pi]$ is an analytic Kramer kernel for the data $\left\{z_{n}\right\}_{n \in \mathbb{Z}}$ and $a_{n}=1$. Thus, for any function $f \in P W_{\pi}$ we have the sampling expansion

$$
f(z)=\sum_{n=-\infty}^{\infty} f\left(z_{n}\right) S_{n}(z)=\sum_{n=-\infty}^{\infty} f\left(z_{n}\right) \frac{P(z)}{\left(z-z_{n}\right) P^{\prime}\left(z_{n}\right)}, \quad z \in \mathbb{C},
$$

where, for each $n \in \mathbb{Z}$, the sampling function $S_{n}(z)=\left\langle K(z), h_{n}\right\rangle_{L^{2}[-\pi, \pi]}, z \in \mathbb{C}$, being $\left\{h_{n}(w)\right\}_{n \in \mathbb{Z}}$ the dual Riesz basis of $\left\{\mathrm{e}^{i z_{n} w} / \sqrt{2 \pi}\right\}_{n \in \mathbb{Z}}$ in $L^{2}[-\pi, \pi]$, and $P$ is an entire function having only simple zeros at $\left\{z_{n}\right\}_{n \in \mathbb{Z}}$. Since a result from Titchmarsh [44] assures that the functions in $P W_{\pi}$ are completely determined by their zeros, we derive that, up to a constant factor, the entire function $P(z)$ coincides with the (convergent) infinite product

$$
\left(z-z_{0}\right) \prod_{n=1}^{\infty}\left(1-\frac{z}{z_{n}}\right)\left(1-\frac{z}{z_{-n}}\right), \quad z \in \mathbb{C} .
$$

Example 2.7. The space $\mathcal{H}_{K_{a}}$ in example 2.5 does not satisfy the $Z R$ property. Indeed, the function $(z-a) \operatorname{sinc} z$ belongs to $\mathcal{H}_{K_{a}}$ since $(z-a) \operatorname{sinc} z=\left\langle K_{a}(z), 1 / 2 \pi\right\rangle_{1}$ for all $z \in \mathbb{C}$. However, by using the sampling formula for $\mathcal{H}_{K_{a}}$ it is straightforward to check that the function $\operatorname{sinc} z$ does not belong to $\mathcal{H}_{K_{a}}$. As a consequence, the sampling formula in example 2.5 cannot be expressed as a Lagrange-type interpolation series.

Example 2.8. Let $K: \mathbb{C} \rightarrow \mathcal{H}$ be an analytic kernel such that $K\left(z_{0}\right)=0$ for some $z_{0} \in \mathbb{C}$. Then all the functions in the associated space $\mathcal{H}_{K}$ have a zero at $z_{0}$ and the $Z R$ property does not hold in $\mathcal{H}_{K}$. Indeed, let $f$ be a nonzero entire function in $\mathcal{H}_{K}$ and let $r$ denote the order of its zero $z_{0}$. The function $f(z) /\left(z-z_{0}\right)^{r}$ is not in $\mathcal{H}_{K}$ since it does not vanish at $z_{0}$.

The kernel $K_{s}: \mathbb{C} \longrightarrow L^{2}[0, \pi]$ defined, for each $z \in \mathbb{C}$, by $\left[K_{s}(z)\right](x)=\sin z x$, $x \in[0, \pi]$ is an analytic Kramer kernel. Any function $f(z)=\langle\sin z x, F\rangle_{L^{2}[0, \pi]}, z \in \mathbb{C}$, where $F \in L^{2}[0, \pi]$, satisfies the sampling expansion

$$
f(z)=\frac{2}{\pi} \sum_{n=1}^{\infty} f(n) \frac{(-1)^{n} n \sin \pi z}{z^{2}-n^{2}}, \quad z \in \mathbb{C} .
$$

Since $K_{s}(0)=0$, the $Z R$ property does not hold and the above sampling formula cannot be written as a Lagrange-type interpolation series.

\section{The semi-inner-product version in Banach spaces}

A semi-inner-product in a Banach space possesses some but not all properties of an inner product. Thus, some Hilbert space arguments and results become available in the presence of a semi-inner-product in a Banach space. The needed preliminaries for this section are taken from [23] and references therein. 


\section{Reproducing kernel Banach spaces}

Following Ref. [49], a reproducing kernel Banach space on $\Omega \subseteq \mathbb{R}$ (or $\mathbb{C}$ ) is a reflexive Banach space $\mathcal{B}$ of functions on $\Omega$ for which its dual space $\mathcal{B}^{*}$ is isometric to a Banach space $\widetilde{\mathcal{B}}$ of functions on $\Omega$ and the point evaluation is continuous on both $\mathcal{B}$ and $\widetilde{\mathcal{B}}$ for each $t \in \Omega$. It has been proved in [49] that there exists a reproducing kernel for an RKBS as defined above. To this end, we introduce the bilinear form on $\mathcal{B} \times \mathcal{B}^{*}$ by setting

$$
\left(u, v^{*}\right)_{\mathcal{B}}:=v^{*}(u), \quad u \in \mathcal{B}, v^{*} \in \mathcal{B}^{*} .
$$

As $\mathcal{B}$ is a reflexive Banach space, then for any bounded linear functional $T$ on $\mathcal{B}^{*}$ there exists a unique $u \in \mathcal{B}$ such that $T\left(v^{*}\right)=\left(u, v^{*}\right)_{\mathcal{B}}$ for each $v^{*} \in \mathcal{B}^{*}$. The following result holds [49, Th. 2]:

Theorem 7. Suppose that $\mathcal{B}$ is an $R K B S$ on $\Omega$. Then there exists a unique function $k: \Omega \times \Omega \longrightarrow \mathbb{C}$ such that the following statements hold:

(a) For every $t \in \Omega, k(\cdot, t) \in \mathcal{B}^{*}$ and $f(t)=(f, k(\cdot, t))_{\mathcal{B}}$ for all $f \in \mathcal{B}$.

(b) For every $t \in \Omega, k(t, \cdot) \in \mathcal{B}$ and $f^{*}(t)=\left(k(t, \cdot), f^{*}\right)_{\mathcal{B}}$ for all $f^{*} \in \mathcal{B}^{*}$.

(c) The linear span of $\{k(t, \cdot): t \in \Omega\}$ is dense in $\mathcal{B}$.

(d) The linear span of $\{k(\cdot, t): t \in \Omega\}$ is dense in $\mathcal{B}^{*}$.

(e) For all $t, s \in \Omega, k(t, s)=(k(t, \cdot), k(\cdot, s))_{\mathcal{B}}$.

The function $k$ in Theorem 7 is the reproducing kernel for the RKBS $\mathcal{B}$. This reproducing kernel is unique. However, as showed in [49], different RKBSs may have the same reproducing kernel: For $1<p<\infty$, the Paley-Wiener classes

$$
\mathcal{B}_{p}:=\left\{f \in C(\mathbb{R}) \mid \operatorname{supp} \widehat{f} \subset[-1 / 2,1 / 2] \text { and } \widehat{f} \in L^{p}[-1 / 2,1 / 2]\right\}
$$

with norm $\|f\|_{\mathcal{B}_{p}}:=\|\widehat{f}\|_{L^{p}[-1 / 2,1 / 2]}$ are RKBSs (not isomorphic), and they all have the function $k(t, s)=\operatorname{sinc}(t-s)$ as the reproducing kernel (here $\left.f(t)=\int_{-1 / 2}^{1 / 2} \widehat{f}(w) \mathrm{e}^{2 \pi \mathrm{i} w t} d w\right)$. In other words, although we have at hand a reproducing kernel $k$, we cannot determine the norm on $\mathcal{B}$. Also, the reproducing kernel for a general RKBS may be an arbitrary function on $\Omega \times \Omega$ which, in particular, might be non-symmetric or non-positive definite [49, Prop. 5]. In order for the reproducing kernel of an RKBS to have the desired properties of that of an RKHS, we impose certain structures on RKBSs, which in some sense are substitutes of the inner product for RKHSs. For this purpose, we adopt the semi-inner-product introduced by Lumer [36] (see also [24, 49]):

\section{Semi-inner-product reproducing kernel Banach space}

Let $\mathcal{B}$ be a Banach space. A semi-inner-product on $\mathcal{B}$ is a function

$$
[\cdot, \cdot]: \mathcal{B} \times \mathcal{B} \longrightarrow \mathbb{C}
$$

such that, for all $x_{1}, x_{2}, x_{3} \in \mathcal{B}$ and $\alpha \in \mathbb{C}$ : 
1. $\left[x_{1}+x_{2}, x_{3}\right]=\left[x_{1}, x_{3}\right]+\left[x_{2}, x_{3}\right]$,

2. $\left[\alpha x_{1}, x_{2}\right]=\alpha\left[x_{1}, x_{2}\right]$ and $\left[x_{1}, \alpha x_{2}\right]=\bar{\alpha}\left[x_{1}, x_{2}\right]$,

3. $\left[x_{1}, x_{1}\right]>0$ for all $x_{1} \neq 0$,

4. $\left|\left[x_{1}, x_{2}\right]\right|^{2} \leq\left[x_{1}, x_{1}\right]\left[x_{2}, x_{2}\right]$.

The difference between a semi-inner-product and an inner product is the conjugate symmetry and, as a consequence, a semi-inner-product may not be additive in the second variable. Every normed vector space $\mathcal{B}$ has a semi-inner-product that induces its norm $[24,36]$, i.e., $\|x\|_{\mathcal{B}}=[x, x]^{1 / 2}$ for each $x \in \mathcal{B}$. In general, a semi-inner-product for a normed vector space may not be unique; however, if the space $\mathcal{B}$ is uniformly Fréchet differentiable we obtain the uniqueness of the semi-inner-product (see [49] for the details). Recall that the space $\mathcal{B}$ is uniformly Fréchet differentiable if for all $x, y \in \mathcal{B}$ with $x \neq 0$, the limit

$$
\lim _{\substack{t \in \mathbb{R} \\ t \rightarrow 0}} \frac{\|x+t y\|_{\mathcal{B}}-\|x\|_{\mathcal{B}}}{t}
$$

exists and it is uniform on $\mathcal{S}(\mathcal{B}) \times \mathcal{S}(\mathcal{B})$ where $\mathcal{S}(\mathcal{B}):=\left\{x \in \mathcal{B}:\|x\|_{\mathcal{B}}=1\right\}$.

Assuming also that the Banach space is uniformly convex we obtain a Riesz representation theorem [24]: For each $f \in \mathcal{B}^{*}$ there exists a unique $x \in \mathcal{B}$ such that $f=x^{*}$. In other words,

$$
f(y)=[y, x]_{\mathcal{B}} \quad \text { for all } y \in \mathcal{B} .
$$

Moreover, $\|f\|_{\mathcal{B}^{*}}=\|x\|_{\mathcal{B}}$. Recall that $\mathcal{B}$ is uniformly convex if for all $\varepsilon>0$ there exists $\delta>0$ such that $\|x+y\|_{\mathcal{B}} \leq 2-\delta$ for all $x, y \in \mathcal{S}(\mathcal{B})$ with $\|x-y\|_{\mathcal{B}} \geq \varepsilon$. Notice that if $\mathcal{B}$ is uniformly convex then it is reflexive (see [39, p.410]) and strictly convex, i.e., for every $x, y \in \mathcal{B}$ with $x \neq y$ and $\|x\|=\|y\|=1$, we have that $\|x+y\|<2$.

For $1<p<\infty$, the classical $L^{p}(I)$, where $I$ denotes any interval on $\mathbb{R}$, and $\ell^{p}(\mathbb{N})$ spaces are uniformly convex and uniformly Fréchet differentiable Banach spaces. Their semi-inner-product are given, respectively, by

$$
[f, g]_{p}:=\|g\|_{p}^{2-p} \int_{I} f(t) \overline{g(t)}|g(t)|^{p-2} d t \quad \text { and } \quad[x, y]_{p}:=\|y\|_{p}^{2-p} \sum_{n=1}^{\infty} x_{n} \overline{y_{n}}\left|y_{n}\right|^{p-2}
$$

We define a semi-inner-product reproducing kernel Banach space (hereafter s.i.p. RKBS) on $\Omega$ as a uniformly convex and uniformly Fréchet differentiable reproducing kernel Banach space on $\Omega$.

An RKHS is an s.i.p. RKBS. Also, the dual of an s.i.p. RKBS remains an s.i.p. RKBS. An s.i.p. RKBS $\mathcal{B}$ has a unique semi-inner-product which represents the interaction between $\mathcal{B}$ and $\mathcal{B}^{*}$. This leads to a more specific representation of the reproducing kernel. Precisely, we have the following result [49, Th. 9]:

Theorem 8. Let $\mathcal{B}$ be an s.i.p. $R K B S$ on $\Omega$ and $k$ its reproducing kernel. Then there exists a unique function $G: \Omega \times \Omega \longrightarrow \mathbb{C}$ such that $\{G(t, \cdot): t \in \Omega\} \subset \mathcal{B}$ and

$$
f(t)=[f, G(t, \cdot)]_{\mathcal{B}} \quad \text { for all } f \in \mathcal{B}, \quad t \in \Omega .
$$


Moreover, there holds the relationship

$$
k(\cdot, t)=(G(t, \cdot))^{*}, \quad t \in \Omega
$$

and

$$
f^{*}(t)=[k(t, \cdot), f]_{\mathcal{B}} \quad \text { for all } f \in \mathcal{B}, \quad t \in \Omega .
$$

We call the unique function $G$ in theorem above the s.i.p. kernel of the s.i.p. RKBS $\mathcal{B}$. It coincides with the reproducing kernel $k$ when $\mathcal{B}$ is an RKHS. In general, when $G=k$ in Theorem 8, we call $G$ an s.i.p. reproducing kernel. Thus, an s.i.p. reproducing kernel $G$ satisfies that $G(t, s)=[G(t, \cdot), G(s, \cdot)]_{\mathcal{B}}, t, s \in \Omega$.

\section{The analogous Kramer sampling result}

Consider a separable complex uniform (i.e., both uniformly Fréchet differentiable and uniformly convex) Banach space $\mathcal{B}$ and denote by $[\cdot, \cdot]_{\mathcal{B}}$ the unique compatible semiinner product on $\mathcal{B}$; let $\mathcal{B}^{*}$ be its dual space. The counterpart to Riesz bases expansions in an s.i.p. RKBS is given by $X_{d}$-Riesz and $X_{d}^{*}$-Riesz bases.

Let $X_{d}$ be a BK-space on $\mathbb{N}$, i.e., a Banach space of sequences $c=\left\{c_{n}\right\}_{n \in \mathbb{N}} \in \mathbb{C}^{\mathbb{N}}$ such that the linear functionals $c \mapsto c_{n}$ are continuous on $X_{d}$ for $n \in \mathbb{N}$. It is known [34] that its dual space $X_{d}^{*}$ is also a BK-space such that the series $\sum_{n=1}^{\infty} c_{n} d_{n}$ converges for every $c \in X_{d}$ and $d \in X_{d}^{*}$. We suppose that if the series above converges for every $c \in X_{d}$, then $d \in X_{d}^{*}$ and if it converges for every $d \in X_{d}^{*}$, then $c \in X_{d}$. We also assume that $X_{d}$ is reflexive, and that the sequence of the canonical unit vectors $\left\{\delta_{n}\right\}_{n=1}^{\infty}$ is a Schauder basis for both $X_{d}$ and $X_{d}^{*}$. An example of such $B K$-spaces is $X_{d}=\ell^{p}(\mathbb{N})$ for $1<p<\infty$; in this case, $X_{d}^{*}=\ell^{q}(\mathbb{N})$ with $1 / p+1 / q=1$.

Let $\left\{x_{n}^{*}\right\}_{n=1}^{\infty} \subset \mathcal{B}^{*}$ be an $X_{d}^{*}$-Riesz basis for $\mathcal{B}^{*}$. This means that

1. $\overline{\operatorname{span}}\left\{x_{n}^{*}: n \in \mathbb{N}\right\}=\mathcal{B}^{*}$.

2. $\sum_{n=1}^{\infty} c_{n} x_{n}^{*}$ converges in $\mathcal{B}^{*}$ for all $c \in X_{d}^{*}$.

3. There exist $0<A \leq B<\infty$ such that

$$
A\|c\|_{X_{d}^{*}} \leq\left\|\sum_{n=1}^{\infty} c_{n} x_{n}^{*}\right\|_{\mathcal{B}^{*}} \leq B\|c\|_{X_{d}^{*}} \text { for all } c \in X_{d}^{*} .
$$

By [50, Thm. 2.15], there exists a unique (dual) $X_{d}$-Riesz basis $\left\{y_{n}\right\}_{n=1}^{\infty}$ for $\mathcal{B}$ such that $\left[y_{m}, x_{n}\right]_{\mathcal{B}}=\delta_{m, n}$ for $m, n \in \mathbb{N}$, and satisfying the expansions:

$$
x=\sum_{n=1}^{\infty}\left[x, x_{n}\right]_{\mathcal{B}} y_{n} \text { for all } x \in \mathcal{B} \text { and } x^{*}=\sum_{n=1}^{\infty}\left[y_{n}, x\right]_{\mathcal{B}} x_{n}^{*} \text { for all } x^{*} \in \mathcal{B}^{*} \text {. }
$$

If the spaces $X_{d}$ and $X_{d}^{*}$ possess the additional property that for all $c \in X_{d}$ and $d \in X_{d}^{*}$ the series $\sum_{n=1}^{\infty} c_{n} d_{n}$ converges absolutely, then the expansions in (13) are unconditionally convergent, i.e., independent of the summation order (see [50, p. 7]). In particular, it is true for $\ell^{p}$-Riesz bases due to Hölder inequality. 
Next, we obtain an s.i.p. RKBS with explicit s.i.p. reproducing kernel where a sampling theory holds: It is the Banach counterpart of the Kramer sampling theorem given in Section 2. Consider a $\mathcal{B}$-valued function $K: \Omega \subset \mathbb{C} \rightarrow \mathcal{B}$ and define, for each $x \in \mathcal{B}$, the function

$$
\begin{aligned}
f_{x}: \Omega & \longrightarrow \mathbb{C} \\
t & \longmapsto[x, K(t)]_{\mathcal{B}} .
\end{aligned}
$$

Then, we have a linear transform $\mathcal{T}_{K}$ on $\mathcal{B}$ with values in $\mathbb{C}^{\Omega}$ such that $\mathcal{T}_{K} x=f_{x}$. Indeed, for $x, y \in \mathcal{B}$ and $\alpha, \beta \in \mathbb{C}$, we have

$$
f_{\alpha x+\beta y}(t)=[\alpha x+\beta y, K(z)]_{\mathcal{B}}=\alpha[x, K(t)]_{\mathcal{B}}+\beta[y, K(t)]_{\mathcal{B}}=\alpha f_{x}(t)+\beta f_{y}(t),
$$

for all $t \in \Omega$.

Having in mind (13), for each $t \in \Omega$, we can write $[K(t)]^{*}=\sum_{n=1}^{\infty}\left[y_{n}, K(t)\right]_{\mathcal{B}} x_{n}^{*}$. We denote $S_{n}(t):=\left[y_{n}, K(t)\right]_{\mathcal{B}}=f_{y_{n}}(t), t \in \Omega$. Suppose that there exists a sequence $\left\{t_{n}\right\}_{n=1}^{\infty}$ in $\Omega$ and $\left\{a_{n}\right\}_{n=1}^{\infty}$ in $\mathbb{C} \backslash\{0\}$ such that the interpolatory condition

$$
S_{n}\left(t_{m}\right)=a_{n} \delta_{n, m},
$$

holds. Then, we have that $\left[K\left(t_{m}\right)\right]^{*}=a_{m} x_{m}^{*}$ and that $\mathcal{T}_{K}$ is one-to-one. Indeed, if $f_{x}(t)=0$ for all $t \in \Omega$,

$$
\begin{aligned}
0=f_{x}(t) & =[x, K(t)]_{\mathcal{B}}=\left[\sum_{n=1}^{\infty}\left[x, x_{n}\right]_{\mathcal{B}} y_{n}, K(t)\right] \\
& =\sum_{n=1}^{\infty}\left[x, x_{n}\right]_{\mathcal{B}}\left[y_{n}, K(t)\right]_{\mathcal{B}}=\sum_{n=1}^{\infty}\left[x, x_{n}\right]_{\mathcal{B}} S_{n}(t),
\end{aligned}
$$

where we have used that $x \mapsto[x, y]_{\mathcal{B}}$ is a continuous functional for any fixed $y \in \mathcal{B}$. Evaluating at $t_{m}$, we have that $\left[x, x_{m}\right]_{\mathcal{B}}=0$ for each $m \in \mathbb{N}$. This implies that $x=0$ and then $\mathcal{T}_{K}$ is one-to-one.

Denote $\mathcal{B}_{K}:=\mathcal{T}_{K}(\mathcal{B})$, the range of the operator $\mathcal{T}_{K}$. If we define $\left\|f_{x}\right\|_{\mathcal{B}_{K}}:=\|x\|_{\mathcal{B}}$ we obtain that $\mathcal{B}_{K}$ is a Banach space of functions defined on $\Omega$ and valued on $\mathbb{C}$. Moreover, $\left[f_{x}, f_{y}\right]_{\mathcal{B}_{K}}:=[x, y]_{\mathcal{B}}$ defines a compatible semi-inner-product on $\mathcal{B}_{K}$. The space $\mathcal{B}_{K}$ becomes an s.i.p. RKBS whose s.i.p. reproducing kernel is given by

$$
k(t, s)=[K(t), K(s)]_{\mathcal{B}}, \quad t, s \in \Omega .
$$

Indeed, for each $t \in \Omega$, the evaluation functional $E_{t}: \mathcal{B}_{K} \longrightarrow \mathbb{C}$ is continuous:

$$
\left|E_{t}\left(f_{x}\right)\right|=\left|f_{x}(t)\right|=\left|[x, K(t)]_{\mathcal{B}}\right| \leq\|x\|_{\mathcal{B}}\|K(t)\|_{\mathcal{B}}=\|K(t)\|_{\mathcal{B}}\left\|f_{x}\right\|_{\mathcal{B}_{K}} .
$$

Observe that, by definition, $k_{t}:=k(t, \cdot)=f_{K(t)} \in \mathcal{B}_{K}$ for all $t \in \Omega$. Hence we deduce that

$$
f_{x}(t)=[x, K(t)]_{\mathcal{B}}=\left[f_{x}, k_{t}\right]_{\mathcal{B}_{K}}=\left[f_{x}, k(t, \cdot)\right]_{\mathcal{B}_{K}},
$$

being $k$ the s.i.p. reproducing kernel for $\mathcal{B}_{K}$. See [49, Th. 10] for more details.

Note also that convergence in the norm of $\mathcal{B}_{K}$ implies pointwise convergence and uniform convergence in subsets of $\Omega$ where the function $t \mapsto\|K(t)\|_{\mathcal{B}}$ is bounded. 
Lemma 9. For every $t \in \Omega$, the sequence $\left\{S_{n}(t)\right\}_{n=1}^{\infty}$ is an element of $X_{d}^{*}$.

Proof. Consider $c \in X_{d}$ and $t \in \Omega$. We must prove that $\sum_{n=1}^{\infty} c_{n} S_{n}(t)$ is convergent. Indeed, by using that for each $z \in \mathcal{B}$ the mapping $x \mapsto[x, z]_{\mathcal{B}}$ is a continuous linear functional on $\mathcal{B}$, and $\left\{y_{n}\right\}_{n=1}^{\infty}$ is an $X_{d^{-}}$Riesz basis for $\mathcal{B}$, we get

$$
\begin{aligned}
\left|\sum_{n=1}^{\infty} c_{n} S_{n}(t)\right| & =\left|\sum_{n=1}^{\infty} c_{n}\left[y_{n}, K(t)\right]_{\mathcal{B}}\right|=\left|\left[\sum_{n=1}^{\infty} c_{n} y_{n}, K(t)\right]_{\mathcal{B}}\right| \\
& \leq\|K(t)\|_{\mathcal{B}}\left\|\sum_{n=1}^{\infty} c_{n} y_{n}\right\|_{\mathcal{B}} \leq B\|K(t)\|_{\mathcal{B}}\|c\|_{X_{d}} .
\end{aligned}
$$

Theorem 10 (A Kramer-type sampling theorem for $\mathcal{B}_{K}$ ). Suppose that, for each $t \in \Omega$, we have the expansion $[K(t)]^{*}=\sum_{n=1}^{\infty} S_{n}(t) x_{n}^{*}$, where $\left\{x_{n}^{*}\right\}_{n=1}^{\infty} \subset \mathcal{B}^{*}$ is an $X_{d}^{*}$-Riesz basis for $\mathcal{B}^{*}$ and $S_{n}(t)=\left[y_{n}, K(t)\right]_{\mathcal{B}}$, being $\left\{y_{n}\right\}_{n=1}^{\infty}$ the dual $X_{d}$-Riesz basis for $\mathcal{B}$ of $\left\{x_{n}^{*}\right\}_{n=1}^{\infty}$. Assume also the existence of sequences $\left\{t_{m}\right\}_{m=1}^{\infty} \subset \mathbb{C}$ and $\left\{a_{m}\right\}_{m=1}^{\infty} \subset \mathbb{C} \backslash\{0\}$ such that the interpolatory condition (14) holds. Then, the sequence $\left\{S_{n}\right\}_{n=1}^{\infty}$ is an $X_{d^{-}}$ Riesz basis for $\mathcal{B}_{K}$ and, for each $f \in \mathcal{B}_{K}$, we have the sampling expansion

$$
f(t)=\sum_{n=1}^{\infty} f\left(t_{n}\right) \frac{S_{n}(t)}{a_{n}}, \quad t \in \Omega .
$$

The convergence of the above series is in the norm of $\mathcal{B}_{K}$, and uniform on subsets of $\Omega$ where the function $t \mapsto\|K(t)\|_{\mathcal{B}}$ is bounded.

Proof. First, we prove that the sequence $\left\{S_{n}\right\}_{n=1}^{\infty}$ is an $X_{d}$-Riesz basis for $\mathcal{B}_{K}$.

1. Consider $t \in \Omega$ and $x \in \mathcal{B}$. Then

$$
f_{x}(t)=[x, K(t)]_{\mathcal{B}}=\left[\sum_{n=1}^{\infty}\left[x, x_{n}\right]_{\mathcal{B}} y_{n}, K(t)\right]_{\mathcal{B}}=\sum_{n=1}^{\infty}\left[x, x_{n}\right]_{\mathcal{B}} S_{n}(t),
$$

hence, $\overline{\operatorname{span}}\left\{S_{n}\right\}_{n=1}^{\infty}=\mathcal{B}_{K}$.

2. Let $c$ be in $X_{d}$. As $\mathcal{T}_{K}$ is an isometry and $\left\{y_{n}\right\}_{n=1}^{\infty}$ is an $X_{d}$-Riesz basis for $\mathcal{B}$,

$$
\left\|\sum_{n=1}^{\infty} c_{n} S_{n}\right\|_{\mathcal{B}_{K}}=\left\|\sum_{n=1}^{\infty} c_{n} y_{n}\right\|_{\mathcal{B}},
$$

and thus the series $\sum_{n=1}^{\infty} c_{n} S_{n}$ converges in $\mathcal{B}_{K}$ for each $c \in X_{d}$.

3. As $\mathcal{T}_{K}$ is an isometry, for every $c \in X_{d}$,

$$
A\|c\|_{X_{d}} \leq\left\|\sum_{n=1}^{\infty} c_{n} y_{n}\right\|_{\mathcal{B}}=\left\|\sum_{n=1}^{\infty} c_{n} S_{n}\right\|_{\mathcal{B}_{K}} \leq B\|c\|_{X_{d}} .
$$


Now consider $m \in \mathbb{N}$; we have that

$$
f_{x}\left(t_{m}\right)=\left[x, K\left(t_{m}\right)\right]_{\mathcal{B}}=\sum_{n=1}^{\infty}\left[x, x_{n}\right]_{\mathcal{B}} S_{n}\left(t_{m}\right)=a_{m}\left[x, x_{m}\right]_{\mathcal{B}} .
$$

Thus,

$$
f_{x}(t)=\sum_{n=1}^{\infty}\left[x, x_{n}\right]_{\mathcal{B}} S_{n}(t)=\sum_{n=1}^{\infty} \frac{f_{x}\left(t_{n}\right)}{a_{n}} S_{n}(t),
$$

in the norm of $\mathcal{B}_{K}$. The pointwise and uniform convergence comes from the fact that $\mathcal{B}_{K}$ is an s.i.p. RKBS.

Example 3.1. Consider $p \in(1,2]$ and its conjugate index $q \in \mathbb{R}$, i.e., $1 / p+1 / q=1$. We consider the compatible semi-inner-product for $\mathcal{B}:=L^{p}[-1 / 2,1 / 2]$ given by

$$
[f, g]_{p}:=\|g\|_{p}^{2-p} \int_{-1 / 2}^{1 / 2} f(x) \overline{g(x)}|g(x)|^{p-2} d x .
$$

Remember that $\mathcal{B}^{*}=L^{q}[-1 / 2,1 / 2]$. We take $X_{d}:=\ell^{q}(\mathbb{Z})$, then, $X_{d}^{*}=\ell^{p}(\mathbb{Z})$.

Define $e_{n}(w):=\mathrm{e}^{2 \pi \mathrm{in} w}$ for $n \in \mathbb{Z}$. Easy computations show that $\left\|e_{n}\right\|_{p}=1$. On the other hand, by [49], we have that $e_{n}^{*}(w)=\mathrm{e}^{-2 \pi \mathrm{inw}}$ and that $\left\|e_{n}^{*}\right\|_{q}=\left\|e_{n}\right\|_{p}=1$. We know that (see [46, p. 20]):

$$
\overline{\operatorname{span}}\left\{\mathrm{e}^{-2 \pi \mathrm{inw}}: n \in \mathbb{Z}\right\}=L^{q}[-1 / 2,1 / 2] .
$$

We define the linear operator

$$
\begin{aligned}
U: L^{p}[-1 / 2,1 / 2] & \longrightarrow \mathbb{C}^{\mathbb{Z}} \\
F & \longmapsto\left\{\left[F, e_{n}\right]_{p}\right\}_{n \in \mathbb{Z}}
\end{aligned}
$$

The Hausdorff-Young theorem (see [51, p.101]) ensures that $U$ is a bounded operator with values on $\ell^{q}(\mathbb{Z})$ (and thus a closed operator). We have that $U\left(L^{p}[-1 / 2,1 / 2]\right)$ is a closed subspace of $\ell^{q}(\mathbb{Z})$ and thus, a Banach space with the metric induced by $\ell^{q}(\mathbb{Z})$. Remember that $\left\{\delta_{n}\right\}_{n \in \mathbb{Z}}$ where $\delta_{n}(m)=0$ if $n \neq m$ and $\delta_{n}(n)=1$, is a Schauder basis for $\ell^{q}(\mathbb{Z})$. Thus, as $\delta_{n}=U\left(e_{n}\right)$, we obtain that $U$ is a surjective mapping. By using [50, Prop. 2.12], the sequence $\left\{e_{n}^{*}\right\}_{n \in \mathbb{Z}}$ is an $\ell^{p}(\mathbb{Z})$-Riesz basis for $\mathcal{B}^{*}=L^{q}[-1 / 2,1 / 2]$.

Now, we define $K(z):=\mathrm{e}^{2 \pi \mathrm{i} z \cdot} \in L^{p}[-1 / 2,1 / 2]$ for every $z \in \mathbb{C}$. Thus, we obtain the following s.i.p. RKBS

$$
\mathcal{B}_{K}:=\left\{f(z)=\left[F, \mathrm{e}^{2 \pi \mathrm{i} z w}\right]_{p}, z \in \mathbb{C}, \text { where } F \in L^{p}[-1 / 2,1 / 2]\right\},
$$

endowed with the norm $\|f\|_{\mathcal{B}_{K}}:=\|F\|_{L^{p}[-1 / 2,1 / 2]}$.

Next, we compute $S_{n}(z):=\left[e_{n}, K(z)\right]_{p}$. First, observe that, if we write $z=x+\mathrm{i} y$,

$$
\|K(z)\|_{p}=\left(\int_{-1 / 2}^{1 / 2}\left|\mathrm{e}^{2 \pi \mathrm{i} z w}\right|^{p} d w\right)^{1 / p}=\left(\int_{-1 / 2}^{1 / 2} \mathrm{e}^{-2 \pi y w p} d w\right)^{1 / p}=\operatorname{sinc}^{1 / p}(\mathrm{i} y p) .
$$


Thus, we have that

$$
\begin{aligned}
S_{n}(z) & =\left[e_{n}, K(z)\right]_{p}=\operatorname{sinc}^{(2-p) / p}(\mathrm{i} y p) \int_{-1 / 2}^{1 / 2} \mathrm{e}^{2 \pi \mathrm{i} n w} \mathrm{e}^{-2 \pi \mathrm{i} z w} \mathrm{e}^{-2 \pi y w(p-2)} d w \\
& =\operatorname{sinc}^{(2-p) / p}(\mathrm{i} y p) \int_{-1 / 2}^{1 / 2} \mathrm{e}^{-2 \pi \mathrm{i}[(z-n)-\mathrm{i} y(p-2)] w} d w \\
& =\operatorname{sinc}^{(2-p) / p}(\mathrm{i} y p) \operatorname{sinc}[(z-n)-\mathrm{i} y(p-2)], \quad z \in \mathbb{C},
\end{aligned}
$$

for $n \in \mathbb{Z}$. Moreover, $S_{n}(m)=\delta_{m, n}$ for every $m, n \in \mathbb{Z}$.

Finally, Theorem 10 gives the following sampling formula for any $f \in \mathcal{B}_{K}$ :

$$
f(z)=\operatorname{sinc}^{(2-p) / p}(\mathrm{i} y p) \sum_{n=-\infty}^{\infty} f(n) \operatorname{sinc}[(z-n)-\mathrm{i} y(p-2)], \quad z=x+\mathrm{i} y \in \mathbb{C} .
$$

The convergence of the series in $(15)$ is uniform on horizontal strips of $\mathbb{C}$. Observe that, if $p=2$ or $z \in \mathbb{R}$, formula (15) coincides with the cardinal series.

For further information on the recent topic of sampling in Banach spaces, see, for instance, Refs. [2, 10, 25, 26, 38, 50].

\section{The distributional version}

Following Ref. [17], a Hilbert space $\mathcal{H}$ whose elements are distributions supported on the interval $I=[0,1]$ and for which the natural injection from $\mathcal{H}$ into $\mathcal{E}^{\prime}(\mathbb{R})$ (the space of compact supported distributions) is continuous is called a Hilbert space of distributions on $I$. In general, it is clear that a Hilbert space of distributions on $I$ having the system $\left\{\mathrm{e}^{2 \pi i n x} \chi_{I}(x)\right\}_{n \in \mathbb{Z}}$ as a complete orthogonal system is completely determined by the sequence of positive numbers $c_{n}=\left\|\mathrm{e}^{2 \pi \mathrm{i} n x} \chi_{I}(x)\right\|_{\mathcal{H}}, n \in \mathbb{Z}$. In [17], it is proved that if the sequence $\left\{c_{n}^{-1 / 2} \mathrm{e}^{2 \pi \mathrm{in} x} \chi_{I}(x)\right\}_{n \in \mathbb{Z}}$ is a complete orthonormal system for a Hilbert space $\mathcal{H}$ of distributions on $I$, then we have

$$
\sum_{n \in \mathbb{Z}} \frac{1}{c_{n}\left(1+n^{2}\right)}<\infty
$$

and the inner product in $\mathcal{H}$ is given by

$$
\langle T, S\rangle_{\mathcal{H}}=\sum_{n \in \mathbb{Z}} c_{n} \widehat{T}(n) \overline{\widehat{S}(n)}, \quad T, S \in \mathcal{H}
$$

where $\widehat{T}$ and $\widehat{S}$ are, respectively, the distributional Fourier transforms of $T$ and $S$. Furthermore, since

$$
\left\langle T, c_{n}^{-1 / 2} \mathrm{e}^{2 \pi \mathrm{in} x} \chi_{I}(x)\right\rangle_{\mathcal{H}}=c_{n}^{1 / 2} \widehat{T}(n) \quad \text { for all } T \in \mathcal{H},
$$

any $T \in \mathcal{H}$ can be expanded as

$$
T=\sum_{n \in \mathbb{Z}} \widehat{T}(n) \mathrm{e}^{2 \pi \mathrm{i} n x} \chi_{I}(x),
$$


where the series converges both in the Hilbert space of distributions $\mathcal{H}$, and in $\mathcal{E}^{\prime}(\mathbb{R})$ (see [17] for the details).

Assume that $\left\{T_{n}:=c_{n}^{-1 / 2} \mathrm{e}^{2 \pi i n x} \chi_{I}(x)\right\}_{n \in \mathbb{Z}}$ is a complete orthonormal system for a Hilbert space $\mathcal{H}$ of distributions on $I$. Since the embedding of $\mathcal{H}$ into $\mathcal{E}^{\prime}(\mathbb{R})$ is continuous, for each test function $\phi \in \mathcal{D}(\mathbb{R})$ (the vector space of all complex-valued functions that are infinitely smooth and zero outside some finite interval endowed with its usual topology) there exists a constant $C_{\phi} \geq 0$ such that $|T(\phi)| \leq C_{\phi}\|T\|_{\mathcal{H}}$, for any $T$ in $\mathcal{H}$. In particular, if we choose $\phi_{t}(x)=\mathrm{e}^{-2 \pi \mathrm{itx}}$ on a neighborhood of $I$ then, for every $t \in \mathbb{R}$, there exists a constant $C_{t}$ such that $|\widehat{T}(t)| \leq C_{t}\|T\|_{\mathcal{H}}$ for any $T$ in $\mathcal{H}$, where $\widehat{T}$ denotes the distributional Fourier transform of $T$. This implies that, for every $t \in \mathbb{R}$, the linear functional $T \longmapsto \widehat{T}(t)$ is continuous in $\mathcal{H}$. As a consequence, there exists an element $K(t)$ in $\mathcal{H}$ such that

$$
\widehat{T}(t)=\langle T, K(t)\rangle_{\mathcal{H}}, \quad t \in \mathbb{R} .
$$

Having in mind (17), for every $n \in \mathbb{Z}$ we have that $K(n)=c_{n}^{-1 / 2} T_{n}$. Besides, an easy calculation shows that

$$
S_{n}(t):=\left\langle T_{n}, K(t)\right\rangle_{\mathcal{H}}=\widehat{T_{n}}(t)=c_{n}^{-1 / 2} \mathrm{e}^{-\mathrm{i} \pi(t-n)} \frac{\sin \pi(t-n)}{\pi(t-n)}, \quad t \in \mathbb{R} .
$$

Besides, the function $t \mapsto\|K(t)\|=\sup _{\|T\|=1}|\widehat{T}(t)|$ is bounded on compact subsets of $\mathbb{R}$. Indeed, by applying both sides of $(18)$ on the test function $\mathrm{e}^{-2 \pi \mathrm{i} t x}$ it follows that

$$
\widehat{T}(t)=\sum_{n \in \mathbb{Z}} \widehat{T}(n) \frac{1-\mathrm{e}^{-2 \pi \mathrm{i} t}}{2 \pi \mathrm{i}(t-n)}=\sum_{n \in \mathbb{Z}} c_{n}^{-1 / 2} \widehat{T}(n) \frac{1-\mathrm{e}^{-2 \pi \mathrm{i} t}}{c_{n}^{-1 / 2} 2 \pi \mathrm{i}(t-n)}, \quad t \in \mathbb{R} .
$$

The boundedness on compact subsets of $\mathbb{R}$ is deduced by using Cauchy-Schwarz inequality and taking into account that $\|T\|^{2}=\sum_{n \in \mathbb{Z}} c_{n}|\widehat{T}(n)|^{2}$ and (16).

According to Theorem 1, the corresponding sampling theorem for the RKHS $\mathcal{H}_{K}:=$ $\mathcal{F}(\mathcal{H})$, where $\mathcal{F}$ denotes the distributional Fourier transform, reads as follows:

Example 4.1. Let $\mathcal{H}$ be a Hilbert space of distributions on $I=[0,1]$ such that the sequence $\left\{c_{n}^{-1 / 2} \mathrm{e}^{2 \pi \mathrm{i} n x} \chi_{I}(x)\right\}_{n \in \mathbb{Z}}$ is a complete orthonormal system. Then, any function $g \in \mathcal{H}_{K}$, i.e., $g(t)=\langle T, K(t)\rangle_{\mathcal{H}}, t \in \mathbb{R}$, for some $T \in \mathcal{H}$, can be recovered from its samples at $\mathbb{Z}$ by means of the sampling formula

$$
g(t)=\sum_{n \in \mathbb{Z}} c_{n}^{1 / 2} g(n) S_{n}(t)=\sum_{n \in \mathbb{Z}} g(n) \mathrm{e}^{-\mathrm{i} \pi(t-n)} \frac{\sin \pi(t-n)}{\pi(t-n)}, \quad t \in \mathbb{R} .
$$

The series converges in the $\mathcal{H}_{K}$-norm sense and also absolutely and uniformly on compact subsets of $\mathbb{R}$.

\section{Hilbert spaces of distributions having a reproducing distribution}

A distributional version of Kramer sampling theorem can be stated in terms of Hilbert spaces of distributions having a reproducing distribution. Let $\mathcal{D}:=\mathcal{D}(\mathbb{R})$ be the vector space of test functions, let $\mathcal{H}$ be a separable Hilbert space and let $K: \mathcal{D} \rightarrow \mathcal{H}$ be a 
continuous conjugate linear function. In particular, whenever $\mathcal{H}=L^{2}(\mathbb{R})$ the conjugate map $\bar{K}$ is just a generalized stochastic process in the sense of $[12,37]$. For any $x \in \mathcal{H}$ the function $h_{x}(\phi):=\langle x, K(\phi)\rangle_{\mathcal{H}}$ is well-defined as a function $h_{x}: \mathcal{D} \rightarrow \mathbb{C}$. Moreover, $h_{x}$ is a continuous linear form because $K$ is continuous and conjugate linear. Denote by $\mathcal{H}_{K} \subset \mathcal{D}^{\prime}$ the set of distributions obtained in this way and by $\mathcal{T}_{K}$ the linear transform

$$
\mathcal{T}_{K}: \mathcal{H} \ni x \longmapsto h_{x} \in \mathcal{H}_{K}
$$

Note that $\mathcal{T}_{K}: \mathcal{H} \longrightarrow \mathcal{D}^{\prime}$ is the transpose of the function $K$. If we endow $\mathcal{H}_{K}$ with the norm $\|h\|_{\mathcal{H}_{K}}=\inf \left\{\|x\|_{\mathcal{H}}: h=\mathcal{T}_{K}(x)\right\}$, we obtain a reproducing distribution Hilbert space whose reproducing distribution is given by, cf. [12, p. 40],

$$
k(\phi, \psi)=\langle K(\psi), K(\phi)\rangle_{\mathcal{H}}, \quad \phi, \psi \in \mathcal{D},
$$

i.e., for each $\psi \in \mathcal{D}$ the function $k_{\psi}$ defined as $k_{\psi}(\phi):=k(\phi, \psi)$ belongs to $\mathcal{H}_{K}$, and the reproducing property

$$
h(\psi)=\left\langle h, k_{\psi}\right\rangle_{\mathcal{H}_{K}}=\langle h, k(\cdot, \psi)\rangle_{\mathcal{H}_{K}}, \quad \psi \in \mathcal{D}, \quad h \in \mathcal{H}_{K}
$$

holds. Note that the space $\mathcal{H}_{K}$ is nothing but the completion of the range space $\mathcal{T}_{K} K(\mathcal{D})$ which is a pre-Hilbert space with the norm defined by $\|h\|=\|K(\phi)\|_{\mathcal{H}}$ where $\phi \in \mathcal{D}$ is such that $h=\mathcal{T}_{K} K(\phi)$ (see [37]). As for a functional RKHS, the linear operator $\mathcal{T}_{K}$ is one-to-one if and only if the set $\{K(\phi)\}_{\phi \in \mathcal{D}}$ is complete in $\mathcal{H}$.

Note that, in particular, a continuous $\mathcal{H}$-valued function $K: \mathbb{R} \rightarrow \mathcal{H}$ can be considered as a continuous conjugate linear function $\mathbf{K}: \mathcal{D} \rightarrow \mathcal{H}$ through the formula

$$
\mathbf{K}(\phi)=\int_{-\infty}^{\infty} \overline{\phi(t)} K(t) d t
$$

The (Bochner) integral in this formula has always a meaning because for every fixed $\phi \in$ $\mathcal{D}$ the integral is defined on a set of finite measure, the $\mathcal{H}$-valued function $\bar{\phi} K$ is weakly Lebesgue measurable and then strongly Lebesgue measurable since $\mathcal{H}$ is separable, and $\int_{-\infty}^{\infty}\|\overline{\phi(t)} K(t)\|_{\mathcal{H}} d t<\infty$. Thus, the distributional version is indeed a generalization of the RKHS setting of Section 2.

The corresponding Kramer sampling theorem for distributions in $\mathcal{H}_{K}$ reads as follows:

Theorem 11. Assume that there exist $\left\{\phi_{n}\right\}_{n=1}^{\infty} \in \mathcal{D},\left\{a_{n}\right\}_{n=1}^{\infty} \in \mathbb{C} \backslash\{0\}$ such that $K\left(\phi_{n}\right)=\bar{a}_{n} x_{n}$ for some Riesz basis $\left\{x_{n}\right\}_{n=1}^{\infty}$ for $\mathcal{H}$. Then, the sequence of distributions $\left\{S_{n}\right\}_{n=1}^{\infty}$ given by

$$
S_{n}(\phi):=\left\langle y_{n}, K(\phi)\right\rangle_{\mathcal{H}}, \quad \phi \in \mathcal{D}, \quad n \in \mathbb{N},
$$

where $\left\{y_{n}\right\}_{n=1}^{\infty}$ is the dual base of $\left\{x_{n}\right\}_{n=1}^{\infty}$, forms a Riesz basis for the space $\mathcal{H}_{K}$. Expanding any $h \in \mathcal{H}_{K}$ in this Riesz basis we obtain

$$
h(\phi)=\sum_{n=1}^{\infty} h\left(\phi_{n}\right) \frac{S_{n}(\phi)}{a_{n}}, \quad \phi \in \mathcal{D} .
$$

The series in (22) converges in the $\mathcal{H}_{K}$-norm sense and also, absolutely and uniformly on subsets of $\mathcal{D}$ where $\phi \mapsto\|K(\phi)\|_{\mathcal{H}}$ is bounded. 
Proof. It is similar to the proof of Theorem 1: From $K\left(\phi_{n}\right)=\bar{a}_{n} x_{n}$ we deduce that $\mathcal{T}_{K}$ is a bijective isometry and since $\mathcal{T}_{K}\left(y_{m}\right)=S_{m}, m \in \mathbb{N}$, we obtain that $\left\{S_{m}\right\}_{m=1}^{\infty}$ is a Riesz basis for $\mathcal{H}_{K}$ whose biorthonormal basis $\left\{T_{m}\right\}_{m=1}^{\infty}$ is given by $T_{m}=\mathcal{T}_{K}\left(x_{m}\right)$, $m \in \mathbb{N}$. Expanding any $h \in \mathcal{H}_{K}$ in this Riesz basis, we have $h=\sum_{n=1}^{\infty}\left\langle h, T_{n}\right\rangle_{\mathcal{H}_{K}} S_{n}$, in the $\mathcal{H}_{K}$-norm sense and, consequently, pointwise in $\mathcal{D}$ since for any $\phi \in \mathcal{D}$ the mapping $h \mapsto h(\phi)$ is continuous on $\mathcal{H}_{K}\left[12\right.$, p. 41]. Moreover, having in mind that $\mathcal{T}_{K}$ is an isometry, and that $K\left(\phi_{n}\right)=\bar{a}_{n} x_{n}$ we obtain

$$
\left\langle h, T_{n}\right\rangle_{\mathcal{H}}=\left\langle\mathcal{T}_{K}(x), \mathcal{T}_{K}\left(x_{n}\right)\right\rangle_{\mathcal{H}_{K}}=\left\langle x, x_{n}\right\rangle_{\mathcal{H}}=\left\langle x, K\left(\phi_{n}\right) / \bar{a}_{n}\right\rangle_{\mathcal{H}}=h\left(\phi_{n}\right) / a_{n}, \quad n \in \mathbb{N},
$$

and hence the sampling expansion (22). As in Theorem 1, the absolute convergence of the series on each $\phi \in \mathcal{D}$ follows from its unconditionally convergence and the uniform convergence on subsets of $\mathcal{D}$ where $\phi \mapsto\|K(\phi)\|_{\mathcal{H}}$ is bounded follows by using CauchySchwarz's inequality in the reproducing property (21).

Example 4.2. Let $\left\{e_{n}\right\}_{n \in \mathbb{Z}}$ be an orthonormal basis of a separable Hilbert space $\mathcal{H}$ and let a non zero function $\phi_{0} \in \mathcal{D}$ such that $\operatorname{supp} \phi_{0} \subset I$ where $I \subset \mathbb{R}$ is an arbitrary interval of length $l(I) \leq 1$. For every $n \in \mathbb{Z}$, set $\phi_{n}(t):=\phi_{0}(t-n), \quad t \in \mathbb{R}$, and consider the function $K: \mathcal{D} \rightarrow \mathcal{H}$ defined by

$$
K(\psi)=\sum_{n \in \mathbb{Z}}\left\langle\phi_{n}, \psi\right\rangle_{L^{2}} e_{n}, \quad \psi \in \mathcal{D} .
$$

Note that for every fixed $\psi \in \mathcal{D}$ there are only a finite set of non-zero addends in the above summation, so that $K(\psi) \in \mathcal{H}$ and it is easily tested that $K$ is an $\mathcal{H}$-valued continuous conjugate linear function. Moreover,

$$
K\left(\phi_{m}\right)=\sum_{n \in \mathbb{Z}}\left\langle\phi_{n}, \phi_{m}\right\rangle_{L^{2}} e_{n}=\left\|\phi_{m}\right\|_{L^{2}}^{2} e_{m}=\left\|\phi_{0}\right\|_{L^{2}}^{2} e_{m}
$$

In order to apply Theorem 11 we compute $S_{m}(\psi)$ :

$$
S_{m}(\psi)=\left\langle e_{m}, K(\psi)\right\rangle_{\mathcal{H}}=\left\langle e_{m}, \sum_{n \in \mathbb{Z}}\left\langle\phi_{n}, \psi\right\rangle_{L^{2}} e_{n}\right\rangle_{\mathcal{H}}=\left\langle\psi, \phi_{m}\right\rangle_{L^{2}} .
$$

Therefore, for every $h \in \mathcal{H}_{K}$ the sampling expansion (22) reads as

$$
h(\psi)=\frac{1}{\left\|\phi_{0}\right\|_{L^{2}}^{2}} \sum_{n \in \mathbb{Z}} h\left(\phi_{n}\right)\left\langle\psi, \phi_{n}\right\rangle_{L^{2}}, \quad \psi \in \mathcal{D},
$$

where the series converges in the $\mathcal{H}_{K}$-norm sense and also, absolutely and uniformly on subsets of $\mathcal{D}$ where $\psi \mapsto \sum_{n \in \mathbb{Z}}\left|\left\langle\phi_{n}, \psi\right\rangle_{L^{2}}\right|^{2}$ is bounded.

Acknowledgments: The authors wish to thank the referees for their valuable and constructive comments. This work has been supported by the grant MTM2009-08345 from the Spanish Ministerio de Ciencia e Innovación (MICINN). 


\section{References}

[1] N. I. Akhiezer. The classical moment problem. Oliver \& Boyd, Edinburgh, 1965.

[2] A. Aldroubi, Q. Sun and W. Tang. $p$-frames and shift-invariant subspaces of $L^{p}$. J. Fourier Anal. Appl., 7(1):1-22, 2001.

[3] N. Aronszajn. Theory of reproducing kernels. Trans. Amer. Math. Soc., 68:337404, 1950.

[4] C. Berg. Indeterminate moment problex and the theory of entire functions. $J$. Comput. Appl. Math., 65:27-55, 1995.

[5] C. Berg and G. Valent. Nevanlinna extremal measures for some orthogonal polynomials related birth and death processes. J. Comput. Appl. Math., 57:29-43, 1995.

[6] A. Boumenir and A. Zayed. The equivalence of Kramer and Shannon sampling theorems revisited. Sampl. Theory Signal Image Process.,4:251-269, 2005.

[7] P. L. Butzer. A survey of the Whittaker-Shannon sampling theorem and some of its extensions. J. Math. Res. Expos., 3:185-212, 1983.

[8] P. L. Butzer and G. Nasri-Roudsari. Kramer sampling theorem and its role in mathematics. In Image Processing: Mathematical Methods and Applications, Oxford University Press, Oxford, UK, 1997, pp. 49-95.

[9] L. Campbell. A comparison of the sampling theorems of Kramer and Whittaker. J. Siam, 12:117-130, 1964.

[10] J. G. Christensen. Sampling in reproducing kernel Banach spaces on Lie groups. J. Approx. Theory, 164(1):179-203, 2010.

[11] O. Christensen. An Introduction to Frames and Riesz Bases. Birkhäuser, Boston, 2003.

[12] R. Cristescu and G. Marinescu. Applications of the Theory of Distributions. John Wiley \& Sons, London, 1973.

[13] W. N. Everitt and G. Nasri-Roudsari. Interpolation and sampling theories, and linear ordinary boundary value problems. In J. R. Higgins and R. L. Stens, eds., Sampling Theory in Fourier and Signal Analysis: Advanced Topics. Oxford University Press, Oxford, 1999, pp. 96-129.

[14] W. N. Everitt, A. G. García and M. A. Hernández-Medina. On Lagrange-type interpolation series and analytic Kramer kernels. Results Math., 51:215-228, 2008.

[15] P. E. Fernández-Moncada, A. G. García and M. A. Hernández-Medina. The zeroremoving property and Lagrange-type interpolation series. Numer. Funct. Anal. Optim., 32:858-876, 2011. 
[16] H. R. Fernández-Morales, A. G. García and G. Pérez-Villalón. Generalized sampling in $L^{2}\left(\mathbb{R}^{d}\right)$ shift-invariant subspaces with multiple stable generators. In Multiscale Signal Analysis and Modeling, pp. 51-80, Lecture Notes in Electrical Engineering, Springer, New York, 2012.

[17] J. P. Gabardo. Hilbert spaces of distributions having an orthogonal basis of exponentials. J. Fourier Anal. Appl., 6(3):277-298, 2000.

[18] A. G. García. Orthogonal sampling formulas: a unified approach. Siam Rev., 42: 499-512, 2000.

[19] A. G. García and M. A. Hernández-Medina. Sampling theorems and difference Sturm-Liouville problems. J. Differ. Equations Appl., 6: 695-717, 2000.

[20] A. G. García and M. A. Hernández-Medina. The discrete Kramer sampling theorem and indeterminate moment problems. J. Comput. Appl. Math., 134:13-22, 2001.

[21] A. G. García and L. L. Littlejohn. On Analytic Sampling Theory. J. Comput. Appl. Math., 171:235-246, 2004.

[22] A. G. García, M. A. Hernández-Medina and F. H. Szafraniec. Analytic Kramer kernels, Lagrange-type interpolation series and de Branges spaces. Complex Var. Elliptic Equ., 58(1):79-97, 2013.

[23] A. G. García and A. Portal. Sampling in reproducing kernel Banach spaces. Mediterr. J. Math., 10(3):1401-1417, 2013.

[24] J. R. Giles. Classes of semi-inner-product spaces. Trans. Amer. Math. Soc., 129: 436-446, 1967.

[25] D. Han and A. Zayed. Sampling expansions for functions having values in a Banach space. Proc. Amer. Math. Soc., 133:3597-3607, 2005.

[26] D. Han, M. Z. Nashed and Q. Sun. Sampling expansions in reproducing kernel Hilbert and Banach spaces. Numer. Funct. Anal. Optim., 30(9-10):971-987, 2009.

[27] G. H. Hardy. Notes on special systems of orthogonal functions, IV: The Whittaker's cardinal series. Proc. Camb. Phil. Soc., 37.331-348, 1941.

[28] J. R. Higgins. Five short stories about the cardinal series. Bull. Amer. Math. Soc., 12:45-89, 1985.

[29] J. R. Higgins. Sampling Theory in Fourier and Signal Analysis: Foundations. Oxford University Press, Oxford, 1996.

[30] J. R. Higgins. A sampling principle associated with Saitoh's fundamental theory of linear transformations. In S. Saitoh et al., eds., Analytic extension formulas and their applications. Kluwer Academic, Dordrecht, 2001, pp. 73-86.

[31] M. E. H. Ismail and D. R. Masson. q-Hermite polynomials, biorthogonal rational functions, and q-beta integrals. Trans. Amer. Math. Soc., 346:63-116, 1994. 
[32] A. J. Jerri. The Shannnon sampling theorem-its various extensions and applications: A tutorial review. Proc. IEEE, 65:1565-1598, 1977.

[33] S. Karlin and J. McGregor. The differential equations of birth and death processes and the Stieltjes moment problem. Trans. Amer. Math. Soc., 85.489-546, 1957.

[34] L. V. Kantorovich and G. P. Akilov. Functional Analysis in Normed Spaces. Macmillan, New York, 1964.

[35] H. P. Kramer. A generalized sampling theorem. J. Math. Phys., 63:68-72, 1959.

[36] G. Lumer. Semi-inner-product spaces. Trans. Amer. Math. Soc., 100:29-43, 1961.

[37] R. Meidan. Reproducing-kernel Hilbert spaces of distributions and generalized stochastic processes. Siam J. Math. Anal., 10(1):62-70, 1979.

[38] M. Z. Nashed and Q. Sun. Sampling and reconstruction of signals in a reproducing kernel subspace of $L^{p}\left(\mathbb{R}^{d}\right)$. J. Funct. Anal., 258:2422-2452, 2010.

[39] W. Rudin. Functional Analysis. McGraw-Hill, 2nd. edition, 1991.

[40] S. Saitoh. Integral Transforms, Reproducing Kernels and Their Applications. Pitman Research Notes in Mathematics Series 369, Longman, Harlow, 1997.

[41] S. Saitoh and Y. Sawano. The theory of reproducing kernels-60 years since N. Aronszajn. Preliminary version, 2013.

[42] B. Simon. The classical moment problem as a self-adjoint finite difference operator. Adv. Math., 137: 82-203, 1998.

[43] A. E. Taylor and D. C. Lay. Introduction to Functional Analysis. John Wiley \& Sons, New York, 1980.

[44] E. C. Titchmarsh. The zeros of certain integral functions. Proc. London Math. Soc., 25:283-302, 1926.

[45] K. Yao. Applications of reproducing kernel Hilbert spaces-bandlimited signal models. Inform. Control, 11:429-444, 1967.

[46] R. M. Young. An Introduction to Nonharmonic Fourier Series. Academic Press, 2001.

[47] P. Weiss. Sampling theorems associated with Sturm-Liouville systems. Bull. Amer. Math. Soc., 63:351, 1957.

[48] A. I. Zayed. Advances in Shannon's Sampling Theory. CRC Press, Boca Raton, 1993.

[49] H. Zhang, Y. Xu, and J. Zhang. Reproducing Kernel Banach Spaces for Machine Learning. J. Mach. Learn. Res., 10:2741-2775, 2009.

[50] H. Zhang and J. Zhang. Frames, Riesz Bases, and Sampling Expansions in Banach Spaces via Semi-inner Products. Appl. Comput. Harmon. Anal., 31:1-25, 2011. 
[51] A. Zygmund. Trigonometric series, Vol. 2. Cambridge University Press, 1959. 